\title{
EL PRINCIPIO DE PROPORCIONALIDAD EN LAS RESTRICCIONES AL DESCUBRIMIENTO PROBATORIO ANTE LA CPI. ESTADO DEL ARTE, CRÍTICAS Y ALTERNATIVAS
}

Simón Moratto*

\begin{abstract}
Resumen: Este texto muestra la función y el desarrollo del principio de proporcionalidad en el establecimiento o autorización de las restricciones al descubrimiento probatorio en la CPI. Para alcanzar este objetivo se estudia el concepto y fundamento normativo de estas figuras en el alto tribunal. Posteriormente, se analizan los puntos de contacto entre las instituciones que aquí convergen con la finalidad de lograr un entendimiento más completo de ellas, de manera que sea posible un análisis crítico del discurso de la Corte. Acto seguido se procede a señalar el estado del arte de este particular uso del principio de proporcionalidad en la jurisprudencia de este tribunal. Finalmente, se presentan algunas problemáticas de las que adolece este desarrollo y se propone una serie de soluciones a ellas.
\end{abstract}

Palabras clave: principio; proporcionalidad; restricciones; descubrimiento probatorio; CPI.

* Abogado por la Universidad Externado de Colombia y magíster en Derecho Penal por la Universidad de Salamanca. Bogotá (Colombia). Correo-e: simonmoratto@gmail.com. Fecha de recepción: 20 de mayo de 2020. Fecha de aceptación: 12 de enero de 2021. Para citar el artículo: Simón MoRATTO. "El principio de proporcionalidad en las restricciones al descubrimiento probatorio ante la CPI. Estado del arte, críticas y alternativas”, Revista Derecho Penal y Criminología, vol. 41, n. ${ }^{\circ} 111$, julio-diciembre de 2020, Bogotá, Universidad Externado de Colombia, pp. 159-205. DoI: https:// doi.org/10.18601/01210483.v41n111.06. 


\title{
THE PRINCIPLE OF PROPORTIONALITY \\ IN THE DISCLOSURE RESTRICTIONS. AT THE ICC. STATE OF THE ART, CRITICISM AND ALTERNATIVES
}

\begin{abstract}
This paper shows the role and development of the principle of proportionality in authorizing disclosure restrictions at the ICC. The concept and normative basis of these figures in this High Court is studied with the aim to achieve this objective. Subsequently, the contact points between these institutions are analyzed in order to get a more complete understanding of them, so that a critical analysis of the Court's discourse is possible. After that, the state of the art about this particular use of the principle of proportionality in the Court's jurisprudence will be indicated. Finally, some issues of this development are presented, and a series of solutions are proposed.
\end{abstract}

Keywords: principle; proportionality; restrictions; disclosure; ICC.

\section{PRINCIPIO DE PROPORCIONALIDAD. CONCEPTO Y FUNDAMENTO NORMATIVO}

\subsection{Concepto del principio de proporcionalidad}

Proporcionalidad significa equilibrio, balanceo, equidad, ponderación, nivelación, igualdad, adecuación ${ }^{1}$, una situación media entre dos posiciones en pugna que en el ámbito del derecho se traducen en intereses jurídicos, los cuales tienen la misma legitimidad para demandar su garantía.

El ordenamiento jurídico está conformado tanto por reglas como por principios. Estos principios, dada la complejidad del sistema normativo y, en general, de la diversidad de las relaciones sociales, suelen colisionar unos con otros, por lo cual no es posible, ni deseable, garantizarles una naturaleza absoluta, sino que deben compaginarse con el objetivo de lograr un sistema ordenado, coherente y justo. En otras palabras, habrá ocasiones en que esos mandatos deban ser limitados y, por tanto, no puede asegurarse su eficacia plena. Un ejemplo claro de un choque entre principios se presenta entre el deber de la fiscalía de buscar la verdad de los hechos y su obligación por el respeto de la integridad del sospechoso en aquellos casos en que se requiere la extracción de una muestra del ADN del procesado con la finalidad de hacer un cotejo probatorio.

1 César Augusto Londoño Ayala. Principio de proporcionalidad en el Derecho procesal penal, Bogotá, Ediciones Nueva Jurídica, 2009, p. 56. 
Las mencionadas restricciones -y es esta la fundamentación en sentido amplio del principio de proporcionalidad en un Estado social de derecho respetuoso de la libertad y la dignidad del ser humano- no pueden ser fruto de un actuar arbitrario de las autoridades estatales, sino que su proceder deber ser razonado. El principio de proporcionalidad, como estructura argumentativa racional, es una herramienta para determinar la legitimidad de las limitaciones a derechos fundamentales y el grado de esa afectación, lo cual cobra especial relevancia en el proceso penal pues es la materia jurídica que en mayor medida y con mayor intensidad puede lesionar estas garantías, dada la incesante tensión entre la protección de los derechos fundamentales y el ejercicio efectivo de la persecución penal ${ }^{2}$.

Ahora bien. Las exigencias mediante las cuales se concreta y estructura el principio de proporcionalidad son tres subprincipios que conforman el llamado "test de proporcionalidad", a saber: subprincipio de idoneidad, subprincipio de necesidad y subprincipio de proporcionalidad en sentido estricto.

En primer lugar, el subprincipio de idoneidad tiene que ver con la optimización de los mandatos dadas las posibilidades fácticas ${ }^{3}$, y se refiere a la causalidad de las medidas en relación con los fines propuestos. Exige una adecuación de los medios con los objetivos desde las perspectivas cualitativa y cuantitativa y de su ámbito subjetivo de aplicación, de tal modo que la transgresión al derecho fundamental facilite la obtención de lo pretendido.

En segundo lugar, el subprincipio de necesidad, que también hace referencia a la pretensión de lograr la mayor realización posible del principio teniendo en cuenta las posibilidades fácticas ${ }^{4}$, alude a que el mecanismo adoptado sea el menos gravoso, en relación con los derechos de quien debe soportarlo, o el único capaz de alcanzar los fines a los que se aspira. En este escenario, se requiere un ejercicio comparativo con otras medidas que sean también idóneas y donde será imperativo un análisis minucioso sobre sus aptitudes y los valores constitucionales en juego ${ }^{5}$.

En tercer lugar, el subprincipio de proporcionalidad stricto sensu expresa la optimización de los mandatos en relación con las posibilidades jurídicas ${ }^{6}$ y ordena la ponderación en el caso concreto de los intereses contrapuestos con el objetivo de corroborar que la medida no genere una carga excesiva para quien debe soportarla.

2 Jaime Bernal Cuéllar y Eduardo Montealegre Lynett. El proceso penal, tomo I: Fundamentos constitucionales y teoría general, Bogotá, Universidad Externado de Colombia, 2013, p. 389.

3 Robert Alexy. Teoría de los derechos fundamentales, Madrid, Centro de Estudios Políticos y Constitucionales, 2007, p. 524.

4 Ibídem.

5 Jaime Bernal Cuéllar y Eduardo Montealegre Lynett. Ob. cit., pp. 427-429.

6 Robert Alexy. Ob. cit., p. 529. 
En otras palabras, debe determinarse si la importancia de la intervención en el derecho fundamental está justificada por la consecución del fin que se pretende, de manera que, posteriormente, sea factible "fundamentar una relación de precedencia entre aquel derecho y este fin"7. Es este el escenario donde se verifica si la afectación del derecho fundamental es intensa, media o leve y a cuál de estas categorías corresponde el beneficio que se obtiene con la medida que restringe el derecho ${ }^{8}$. Este subprincipio, mediante el cual se fija esa relación de precedencia, es idéntico a la ley de la ponderación ${ }^{9}$, una regla argumentativa que define la ponderación en sentido estricto ${ }^{10}$ y en virtud de la cual se determina:

(1) el grado de afectación de uno de los principios;

(2) la importancia de la satisfacción del mandato que juega en contra; y

(3) si la relevancia de la satisfacción de este principio justifica la intervención del primero; en este tercer paso se llega a esa justificación teniendo en cuenta, además del grado de afectación, el peso abstracto ${ }^{11}$ de los mandatos y la seguridad de las apreciaciones empíricas ${ }^{12}$ relativas a la afectación de ellos.

Ahora bien: Alexy complementa la ley de la ponderación con la llamada "fórmula del peso", a través de la cual se articulan la importancia de los principios, su peso abstracto y la seguridad de las apreciaciones empíricas. Se constituye en un recurso metodológico que ilustra la estructura de la ponderación mediante un modelo matemático ${ }^{13}$.

Por último, este decurso argumentativo termina con las denominadas "cargas de la argumentación", las cuales tienen lugar cuando existe un empate resultante de la aplicación de la fórmula del peso ${ }^{14}$.

7 Carlos Bernal Pulido. El principio de proporcionalidad y los derechos fundamentales, Madrid, Centro de Estudios Políticos y Constitucionales, 2007, p. 764.

8 Jaime Bernal Cuéllar y Eduardo Montealegre Lynett. Ob. cit., p. 430.

9 Robert Alexy. Ob. cit., p. 529.

10 Carlos Bernal Pulido. Ob. cit., p. 787.

11 El peso abstracto consiste en que, en ocasiones, dos principios colisionantes tienen la misma jerarquía dada la fuente de derecho que los reconoce pero que, a pesar de esto y según la concepción de los valores predominantes de la sociedad, uno de ellos puede tener mayor importancia in abstracto. Jaime Bernal Cuéllar y Eduardo Montealegre Lynett. El proceso penal, tomo I, cit., p. 408.

12 Las apreciaciones empíricas consisten en la afectación que la medida adoptada proyecta sobre los principios, las cuales pueden tener un mayor o menor grado de certeza y eso significa atribuir un mayor o menor peso al principio en cuestión. Jaime Bernal Cuéllar y Eduardo Montealegre Lynett. El proceso penal, tomo I, cit., pp. 409 y 410.

13 Robert Alexy. "Sobre los derechos constitucionales a protección”, en Robert Alexy, José Bastida Freijedo, Juan Antonio García Amado, Alfonso Jaime García Figueroa, Luis Hierro Sánchez-Pescador, Josep Joan Moreso, C. Pardo, Gregorio Peces-Barba y Ernesto Valdés Garzón. Derechos sociales y ponderación, Madrid, Fundación Coloquio Jurídico Europeo, 2009, pp. 45-84.

14 Aquí Alexy propone dos soluciones contradictorias entre sí. Una donde defiende la existencia de una 


\subsection{Fundamento normativo del principio de proporcionalidad en la CPI}

No existe referencia directa al principio de proporcionalidad en el Estatuto de la Corte ni en las Reglas de Procedimiento y Prueba (en adelante, RPP). Sin embargo, ha manifestado el alto tribunal que se encuentra comprendido en el artículo 81 (4) del Estatuto y en la regla $68(1)^{15}$ de las RPP $^{16}$. Igualmente, debe decirse que tampoco existe una norma que establezca una obligación para los jueces de emplear el test de proporcionalidad.

El hecho de que el principio de proporcionalidad no encuentre consagración directa en la normativa de la Corte Penal Internacional (en adelante, CPI) es una circunstancia común también en los ordenamientos jurídicos nacionales. Este es el caso de España, donde se ha sostenido que este mandato se encuentra implícito en el texto de la Constitución: concretamente, se adscribe al carácter jurídico de los derechos fundamentales, a la idea de justicia, al principio del Estado de derecho y al principio de interdicción de la arbitrariedad ${ }^{17}$.

No obstante, el principio de proporcionalidad cuenta con un reconocimiento prácticamente universal pues es utilizado en casi todas las culturas jurídicas alrededor del mundo ${ }^{18}$. Así mismo, el uso del test de proporcionalidad ha sido ampliamente difundido y ha gozado de gran aceptación global ${ }^{19}$. Incluso algunos tribunales internacionales han vinculado su existencia a otros principios, como la Sala de Primera Instancia (en adelante, SPI) del Tribunal Penal Internacional para la antigua Yugoslavia, la cual sostuvo que la legalidad en la búsqueda de materiales probatorios en posesión del procesado dependía en gran parte de la proporcionalidad de la medida ${ }^{20}$.

carga argumentativa en pro de la libertad y la igualdad jurídica y otra, propuesta quince años después, en virtud de la cual los empates juegan en favor del principio democrático. Para ver en mayor detalle, Robert Alexy. Ob. cit., pp. 59 y ss., y el epílogo a la Teoría de los derechos fundamentales, pp. 511-562.

15 International Criminal Court. Prosecutor v. Thomas Lubanga Dyilo. "Judgment on the Appeal of Mr. Thomas Lubanga Dyilo against the Decision of Pre-Trial Chamber I entitled 'First Decision on the Prosecution Requests and Amended Requests for Redactions under Rule 81 "', ICC-01/04-01/06 (OA 5). 14 December 2006, par. 34.

16 Se resalta que aun cuando el corpus iuris de la CPI no hace referencia directa al principio de proporcionalidad, existen otras normas, además de las indicadas por la Corte, que permiten su desarrollo, verbigracia los artículos 64 (2), 68 (1), 68 (3), 68 (5), 69 (2) del Estatuto y las reglas 84, 91 (3), 101 (1) de las RPP, entre otras disposiciones.

17 Carlos Bernal Pulido. Ob. cit., p. 600.

18 Aharon Barak. Proporcionalidad: los derechos fundamentales y sus restricciones, Lima, Palestra, 2017, pp. 205-243.

19 Ibídem.

20 Conviene mencionar que este tribunal hizo específicamente referencia a los criterios de idoneidad, necesidad y proporcionalidad stricto sensu. International Criminal Tribunal for the Former Yugoslavia (Trial Chamber). Prosecutor v. Milomir Stakic. Order to the Registry of the Tribunal to provide documents. 5 July 2002. 
Sin embargo, y es importante decirlo, a pesar de este antecedente, el requisito de proporcionalidad parece tener una vida muy oscura ${ }^{21}$ en los procesos penales internacionales y las referencias a él no son comunes.

Para finalizar, es necesario decir que ahondar en el fundamento o en la forma en que la CPI ha desarrollado este mandato en su integridad es un tema que supera los límites de esta investigación, de manera que aquí se hará hincapié, únicamente, en su relación con el disclosure en el proceso penal internacional.

\section{DESCUBRIMIENTO DE INFORMACIÓN EN LA CPI. CONCEPTO Y MARCO LEGAL}

\subsection{Concepto de "disclosure"}

El "discovery" es un instituto originado en los sistemas procesales del common law y consiste en permitir a las partes el conocimiento de los elementos materiales probatorios que tengan intención de hacer valer en la presentación de su caso en el juicio. Es oportuno resaltar que el descubrimiento de medios de prueba también es reconocido en procesos de tendencia inquisitiva, aunque de manera indirecta mediante la figura del "case dossier"22. Esta institución también ha recibido el nombre de "disclosure", que describe "the mandatory divulging of information to a litigation opponent according to procedural rules" 23 y cuya naturaleza le hace un elemento esencial en los procesos de tendencia adversarial, constituyéndose en un requisito central para asegurar la eficacia del derecho a un juicio justo.

Aunque discovery y disclosure suelen ser términos que se usan indistintamente, pueden hallarse ciertas diferencias. En Inglaterra y Gales, disclosure hace referencia a la entrega de información relevante de una parte a la otra, mientras que en el sistema judicial de los Estados Unidos se entiende por discovery el momento en el que uno de los contendientes del caso conoce los medios probatorios que la contraparte pretende presentar ${ }^{24}$. En este mismo contexto, se ha definido también como un acto

21 Karel de Meester, Kelly Pitcher, Rod Rastan y Goran Sluiter. "Investigation, Coercive Measures, Arrest and Surrender", en Hakan Friman, Goran Sluiter, Suzannah Linton, Sergey Vasiliev y Salvatore Zappala (eds.). International Criminal Procedure. Principles and Rules, Oxford, Oxford University Press, 2013, pp. 171-381 (traducción propia).

22 Kai Ambos. "Confidential investigations (article 54 (3)(E) ICC Statute) vs. Disclosure obligations: The Lubanga Case and National Law", en New Criminal Law Review: An International and Interdisciplinary Journal, Berkeley, University of California Press, Fall 2009, vol. 12, n. ${ }^{\circ}$ 4, pp. 543-568, disponible en [https://www.legal-tools.org/doc/c76c63/pdf/] (consulta: 7 de julio de 2019).

23 Henry Campbell Black. Black's Law Dictionary. Definitions of the Terms and Phrases of American and English Jurisprudence, Ancient and Modern, 4. ${ }^{\text {a }}$ ed., Minnesota, West Publishing Company, 1968, p. 563.

24 Conforme a esa distinción, puede concluirse que discovery por la defensa corresponde a disclosure 
de la defensa cuyo propósito es obtener información para utilizarla en el juicio, el cual se concreta mediante la petición de producción de documentos, interrogatorios escritos, grabaciones, declaraciones de partes, etc. ${ }^{25}$.

Ahora bien: la razón de ser de esta institución es evitar la introducción de elementos materiales probatorios de manera sorpresiva, previniendo el entorpecimiento del contradictorio, el cual, como se sabe, puede verse fácilmente alterado dada la desventaja natural en la que se encuentra el procesado en relación con la fiscalía ${ }^{26}$. Siguiendo esa lógica, no debe olvidarse que, aunque el procedimiento adquiera una connotación de contienda, "el fin último constitucional del proceso penal es la realización de la justicia material, lo cual implica que el discurso sobre la responsabilidad penal del acusado debe erigirse sobre la base de hechos conocidos y dudas razonables, pero no de pruebas ocultas o acusaciones inesperadas" 27 . En últimas, lo que se quiere eludir es lo que se conoce en el common law como una "emboscada probatoria": "〈no trial by ambush is the underlying rationale of the disclosure obligations, which serves the fairness of the process" 28 .

Para terminar, conviene indicar que la consecuencia de no cumplir con la obligación de descubrir los elementos materiales probatorios, en términos generales, es la incapacidad de estos para convertirse en plena prueba, los cuales, de ser valorados para la toma de una decisión judicial, no pueden acarrear otro resultado que la nulidad del acto por vulneración del derecho a un juicio justo, el principio de igualdad de armas, el principio de contradicción, el derecho de defensa, entre otros. Es más: la gravedad de las conductas puede ser tal que sea necesario ordenar la suspensión o la terminación del proceso, sin perjuicio de las sanciones disciplinarias que consagre el ordenamiento jurídico en cuestión.

\subsection{Marco legal del descubrimiento de información en la Corte Penal Internacional}

El Estatuto de Roma, las RPP y el Reglamento de la Corte son los tres instrumentos principales que regulan los procedimientos ante la CPI. El Estatuto, concretamente, se refiere de manera directa e indirecta al descubrimiento de información. Entre otras

para la fiscalía y discovery por la fiscalía es disclosure para la defensa. Kai Ambos. Treatise on International Criminal Law, Oxford, Oxford University Press, 2013, p. 520.

Kai Ambos. Confidential investigations, cit., p. 561.

Jaime Bernal Cuéllar y Eduardo Montealegre Lynett. El proceso penal, tomo II: Estructura y garantías procesales, 6. ${ }^{a}$ ed., Bogotá, Universidad Externado de Colombia, 2013, p. 771.

7 Corte Constitucional de Colombia. Sentencia C-1194 de 2005, MP: Marco Gerardo Monroy Cabra.

Masha Fedorova. The Principle of Equality of Arms in International Criminal Proceedings, Cambridge, Intersentia, 2013, p. 233. 
cosas, hace referencia a los derechos del acusado ${ }^{29}$, la confirmación de los cargos antes del juicio ${ }^{30}$, las funciones de las salas, los poderes de los jueces ${ }^{31}$ y los deberes $\mathrm{y}$ facultades del fiscal ${ }^{32}$.

En lo que se refiere a las obligaciones en materia de descubrimiento, el Estatuto señala claras diferencias entre fiscalía y defensa, dados los roles que cada parte desempeña ${ }^{33}$. Por su parte, el ente acusador tiene el onus probandi y debe buscar tanto material inculpatorio como exculpatorio, mientras que la labor de la defensa es más bien reactiva a la presentación de medios de prueba por parte del ente persecutor ${ }^{34}$.

Así mismo, el Estatuto y las RPP distinguen dos momentos ${ }^{35}$ en los que tiene lugar la revelación de información. Estas etapas se caracterizan por un alcance diferente del material divulgado: (1) antes de la confirmación de los cargos: donde la presentación del material probatorio tiene por objeto convencer a la Sala de Cuestiones Preliminares (en adelante, SCP) de la existencia de motivos suficientes para creer que la persona investigada cometió cada uno de los cargos alegados; y (2) antes del juzgamiento: donde se allegan potenciales pruebas que se quieren hacer valer en juicio con la finalidad de probar la culpabilidad del procesado más allá de toda duda razonable.

Desde otra perspectiva, se distingue entre el descubrimiento entre las partes propiamente dicho y la obligación de permitir al otro extremo procesal la inspección de libros, fotografías, mapas y objetos tangibles ${ }^{36}$. La primera requiere un rol activo de proporcionar a la contraparte el material probatorio y la segunda hace referencia a una obligación pasiva que ordena permitir el acceso a esos medios de prueba. En esa línea, también se diferencia el descubrimiento que se hace entre las partes y la comunicación de ese material descubierto a la $\mathrm{SCP}^{37}$, la cual determinará si esa actividad tuvo lugar en condiciones satisfactorias.

29 ECPI, artículo 67.

30 ECPI, artículo 61.

31 ECPI, artículo 64.

32 ECPI, artículo 54.

33 International Criminal Court. Prosecutor v. Germain Katanga and Mathieu Ngudjolo Chui. "Decision on the 'prosecution's application concerning disclosure by the defence pursuant to rules 78 and 79 (4)'”, ICC-01/04-01/07. 14 September 2010, par. 36.

34 Ibídem.

35 Christine Schuon. International Criminal Procedure: A Clash of Legal Cultures, The Hague, T. M. C. Asser Press, 2010, p. 275.

36 International Criminal Court. Prosecutor v. Thomas Lubanga Dyilo. "Decision on the final system of disclosure and the establishment of a Timetable", ICC-01/04-01/06. 15 May 2006, p. 4.

37 International Criminal Court. Prosecutor v. Jean Pierre Bemba Gombo. "Decision on the evidence disclosure system and setting a Timetable for disclosure between the parties", ICC-01/05-01/08. 31 July 2008, par. 42. También véase International Criminal Court. Prosecutor v. Thomas Lubanga Dyilo. 
Ahora bien: teniendo en cuenta las normas que regulan la divulgación de información antes de la confirmación de $\operatorname{cargos}^{38}$ y a través de todo el proceso ${ }^{39}$, con el objetivo de lograr un entendimiento general, pero claro, de la estructura del descubrimiento ante la CPI, puede esquematizarse esta institución de la siguiente manera:

\subsubsection{Descubrimiento de información por parte de la fiscalía durante las investigaciones}

Se refiere al material utilizado por el fiscal para solicitar a la SCP la emisión de una orden de arresto. Aquí, se deben ofrecer elementos materiales probatorios lo suficientemente sólidos para creer que la persona cometió un crimen de competencia de la Corte ${ }^{40}$.

\subsubsection{Descubrimiento de información por parte de la} fiscalía antes de la confirmación de los cargos

El propósito de la audiencia de confirmación de cargos es escoger aquellos casos que merecen ir a juicio como resultado de la investigación hecha por el ente persecutor ${ }^{41}$, pero también la posibilidad del procesado de familiarizarse y controvertir tanto los cargos como los medios probatorios presentados para sustentarlos ${ }^{42}$.

Por otro lado, el artículo 61 (3) señala que, en un tiempo razonable antes de la audiencia, la fiscalía debe proveer al investigado una copia del documento que contiene los cargos por los cuales el fiscal le quiere llevar a juicio ${ }^{43}$. Así mismo, este artículo

"Decision on the final system of disclosure and the establishment of a timetable", ICC-01/04-01/06. 15 May 2006, p. 4.

38 ECPI, artículo 63; y RPP-CPI, regla 121.

39 RPP-CPI. Reglas 67, 76, 77 y 83, que señalan la divulgación de elementos probatorios por parte de la fiscalía; y las reglas 78, 79 y 80, que prescriben el descubrimiento por parte de la defensa.

$40 \mathrm{Ni}$ el Estatuto ni las RPP proporcionan información sobre el descubrimiento de medios de prueba a la persona sujeta a una orden de detención antes de entregarse a la Corte; sin embargo, la solución ha sido proporcionada por la jurisprudencia, la cual ha dicho que el descubrimiento puede tener lugar durante la investigación. Véase en mayor detalle Alexander Heinze. International Criminal Procedure and Disclosure: an attempt to better understand and regulate disclosure and communication at the ICC on the basis of a comprehensive and comparative theory of criminal procedure, Berlin, Duncker and Humblot GmbH, 2014, p. 413; International Criminal Court. Prosecutor v. Bemba. "Judgment on the Appeal of Mr. Jean-Pierre Bemba Gombo against the Decision of Pre-Trial Chamber III entitled 'Decision on Application for Interim Release'”, ICC-01/05-01/08-323. 16 December 2008.

41 Alice Chang-Jung Yang. The Prosecution's Duty of Disclosure before International Criminal Tribunals (doctoral thesis), London, Brunel University-Brunel Law School, 2016, p. 303.

42 Ibídem.

43 Una provisión detallada de lo que debe tener este documento se encuentra en el Reglamento de la Corte. Corte Penal Internacional. Magistrados de la Corte, Quinta sesión plenaria, La Haya, 17-28 de mayo de 2004. Documentos oficiales de la Corte Penal Internacional. ICC-BD/01-01-04. Reglamento 
indica que debe ser informado de los medios probatorios en los que la fiscalía basará sus argumentos en la confirmación de cargos.

En ese orden de ideas, la regla 121 (3) complementa el artículo 61 (3) en la medida en que provee un régimen detallado que rige el descubrimiento antes de la confirmación de $\operatorname{cargos}^{44}$. Indica que ese tiempo razonable debe ser de, al menos, treinta días antes de la audiencia, y que la descripción de aquellos, y de los medios de prueba que se pretende usar, debe hacerse de manera detallada. Siguiendo esa línea, y de una lectura conjunta con el artículo 121 (8), se infiere que todo material probatorio allegado después del tiempo límite debe excluirse.

Finalmente, la regla 121 (2) se refiere al deber de comunicar a la SCP todo elemento probatorio descubierto entre las partes. De esta manera, esta sala podrá determinar si ese descubrimiento se realizó en condiciones satisfactorias o no.

\subsubsection{Descubrimiento de información por parte de la fiscalía durante el procedimiento}

La regla 76 consagra la divulgación de información antes del juicio con referencia a los testigos del ente persecutor, el cual está obligado a revelar los nombres de los testigos que quiere llamar a testificar, así como también divulgará sus declaraciones previas en lenguaje original y en un idioma que el sospechoso entienda ${ }^{45}$. Esta obligación debe cumplirse con suficiente antelación para que la defensa pueda preparar su estrategia procesal ${ }^{46}$. Valga mencionar que en la confirmación de cargos la fiscalía no está obligada ${ }^{47}$ a llamar testigos si considera que es suficiente presentar

de la Corte. 26 de mayo de 2004. Norma 52: "El documento en que se formulan los cargos, tal como se menciona en el artículo 61, deberá incluir:

(a) El nombre completo de la persona y cualquier otro dato que sirva para su identificación;

(b) Una relación de los hechos, incluyendo la hora y lugar de los presuntos delitos, que proporcione una base jurídica y fáctica suficiente para hacer que la o las personas comparezcan en juicio, incluyendo hechos pertinentes acerca del ejercicio de su competencia por parte de la Corte;

(c) Una tipificación jurídica de los hechos que dé cuenta tanto de delitos conforme a los artículos 6 , 7 u 8 como de una forma precisa de participación conforme a los artículos 25 y 28 ".

44 Helen Brady. "Disclosure of Evidence", en Roy S. Lee. The International Criminal Court: Elements of Crimes and Rules of Procedure and Evidence, Ardsley, Transnational Publishers, 2001, pp. 403-426.

45 En este contexto puede haber un posible conflicto con la Regla 121 (3) que ordena a la fiscalía proveer una descripción detallada de los cargos y (sólo) una lista (y no ambas, las copias de las declaraciones y los nombres de los testigos que se quieren llamar según lo ordena la regla 76) de los medios de prueba que se quieren hacer valer en la confirmación de cargos. Al respecto, véase Alexander Heinze. Ob. cit., p. 378; Helen Brady. Ob. cit., pp. 403 y 410.

46 El tiempo límite para que decida sobre los medios probatorios en los que pretende confiar es de no menos de treinta días antes de dicha audiencia o quince días en caso de existir nuevos elementos materiales probatorios. International Criminal Court. Prosecutor v. Thomas Lubanga Dyilo. "Decision on the Final System of Disclosure and the Establishment of a Timetable”, ICC-01-04-01-06, par. 105.

47 ECPI, artículo 61 (5). 
documentos o evidencia sumaria ${ }^{48}$. Esta regla se aplica sin perjuicio de las restricciones al descubrimiento que prevén el Estatuto y las RPP $^{49}$.

\subsubsection{Regla 77. Inspección de material probatorio en posesión de la fiscalía}

El fiscal debe permitir la inspección de libros, documentos, fotografías y material tangible que esté en su posesión o bajo su control y que sea material para la preparación de la defensa o que el ente acusador pretende usar en la confirmación de los cargos o en juicio. Igualmente, debe permitirse el acceso a este tipo de material si perteneció o fue obtenido del acusado ${ }^{50}$.

\subsubsection{Descubrimiento de material exculpatorio conforme al artículo 67 (2)}

El fiscal tiene la obligación continua de revelar, tan pronto como sea posible, material exculpatorio que posea. Si el fiscal duda acerca del carácter exculpatorio de la información, será la Corte quien decida si tiene esa calidad o no. Conviene resaltar que este es un deber derivado del artículo 54 que obliga a la fiscalía ${ }^{51}$ a buscar tanto materiales probatorios de cargo como de descargo. Ahora bien: el material que es tanto inculpatorio como exculpatorio debe ser descubierto bajo esta regla ${ }^{52}$.

\subsubsection{Descubrimiento de información por parte de la defensa}

Las reglas 78 y 79 consagran estas obligaciones. La primera disposición señala que la defensa debe permitir la inspección de libros, documentos, fotografías y documentos tangibles que tenga en su posesión o control y que quiera hacer valer en la

48 Sin embargo, si el ente acusador decide confiar en versiones redactadas de declaraciones previas hechas por testigos sin llamarlos, debe proveer copia de las versiones no redactadas de esos testigos a la defensa. Brando Fiori. Disclosure of Information in Criminal Proceedings: A Comparative Analysis of national and international criminal procedural systems and human rights law, Oisterwijk, Wolf Legal Publishers, 2015, p. 261.

49 Restricciones basadas en la necesidad de salvaguardar tanto la protección y privacidad de víctimas y testigos como el resguardo de material confidencial ECPI, artículo 76 (4).

50 Es apropiado mencionar que, dado que esta regla no define lo que debe entenderse por "material para la preparación de la defensa", la Sala de Apelaciones de la CPI ha tenido que interpretarla, y lo ha hecho de manera amplia estableciendo que incluso material que no está directamente relacionado con el caso pero que tiende a explicar la situación general en un escenario de conflicto puede ser "material" en el sentido de la regla 77. Al respecto, véase Alexander Heinze. Ob. cit., p. 355; International Criminal Court. Prosecutor v. Thomas Lubanga Dyilo. "Judgment on the appeals of The prosecutor and The defence against Trial Chamber I's Decision on Victims' participation of 18 January 2008", ICC-01/04-01/06-1432. July 11 2008, pars. 76-82. También véase Cristoph Safferling. International Criminal Procedure, Oxford, Oxford University Press, 2012, p. 278. Algunos autores sostienen que esa amplia interpretación hace que la Regla 121 (3) no tenga sentido. Al respecto, véase Christine Schuon. Ob. cit., p. 278.

51 Alexander Heinze. Ob. cit., p. 344.

52 Alexander Heinze. Ob. cit., p. 347. 
confirmación de cargos o el juicio. El deber de descubrir material por parte de la defensa es más limitado ${ }^{53}$ que el de la fiscalía, estas reglas son permisivas más que obligatorias ${ }^{54}$ y no hay obligaciones a menos que se pretenda usar como evidencia ${ }^{55}$, caso en el cual debe proveer una descripción de los medios probatorios a la SCP dentro de los quince días anteriores a la confirmación de $\operatorname{los} \operatorname{cargos}^{56}$. Esta regla aplica tanto para esta audiencia como para el juicio.

Por otro lado, la regla 79 obliga a la defensa a descubrir a la fiscalía la existencia de una coartada, indicando el lugar donde la persona alega haber estado, así como los nombres de testigos y cualquier otro medio que pretenda usar para fundamentarla. Así mismo, debe descubrir su intención de alegar una causal de exclusión de responsabilidad penal ${ }^{57}$.

Para terminar, la regla 80 señala que debe llevarse a cabo este deber con antelación suficiente para que el ente acusador pueda preparar su caso ${ }^{58}$. No obstante, la regla 79 (3) expresa que fallar en el cumplimiento de esta obligación no limita el derecho de la defensa de hacer valer una coartada o alegar una eximente de responsabilidad penal y presentar medios materiales probatorios con posterioridad.

\subsubsection{Las funciones de la SCP y la SPI en relación con el descubrimiento de información}

La regulación 54 del Reglamento de la $\operatorname{Corte}^{59}$, con el objetivo de que las partes puedan preparase para la audiencia de juzgamiento, establece que la sPI puede ordenar el descubrimiento de medios de prueba y, concretamente, declaraciones de testigos que las partes quieran hacer valer. Así mismo, la regla 84 prescribe que la Sala deberá ${ }^{60}$

53 No obstante, el rol proactivo del juez puede ampliar el alcance de sus obligaciones. Al respecto, véase Brando Fiori. Ob. cit., pp. 268-271.

54 International Criminal Court. Prosecutor v. Thomas Lubanga Dyilo. "Decision on the Disclosure by the Defence", ICC-01/04-01/06. 20 March 2008, par. 27. Ver ECPI, artículo 61 (6).

55 Brando Fiori. Ob. cit., p. 267.

56 RPP-CPI. Regla 121 (6).

57 Estatuto de Roma, artículo 31 (1).

58 El objetivo de esta norma es darle a la fiscalía el contexto en el cual va a desarrollar su interrogatorio de los testigos de la defensa, lo cual permite una mayor eficiencia en los procedimientos, vistos como un todo. International Criminal Court. Prosecutor v. Jean Pierre Bemba Gombo. "Decision on the Defence Disclosure and Related Issues", ICC-01/05-01/08. 24 February 2012, par. 28.

59 Corte Penal Internacional. Magistrados de la Corte, Quinta sesión plenaria, La Haya, 17-28 de mayo de 2004. Documentos oficiales de la Corte Penal Internacional, ICC-BD/01-01-04. Reglamento de la Corte. 26 de mayo de 2004.

60 Con la finalidad, entre otras, de asistir a las partes en la preparación de sus casos, de cumplir con su deber en la búsqueda de la verdad y facilitar un justo y expedito desarrollo de los procedimientos. Brando Fiori. Ob. cit., pp. 271-273; International Criminal Court. Prosecutor v. Thomas Lubanga Dyilo. 
dictar las providencias necesarias para la presentación de pruebas adicionales y para que se descubran documentos o información no revelados previamente ${ }^{61}$. Esta regla concluye prescribiendo que se decretarán fechas estrictas con la finalidad de evitar dilaciones al inicio del juicio.

\subsubsection{El registro de los procedimientos conforme a la regla 121 (10)}

La Secretaría debe crear y mantener un completo y exacto registro de los procedimientos surtidos ante la SCP, el cual debe incluir todo ${ }^{62}$ el material descubierto por las partes y comunicado a la Sala. Este registro estará sujeto a las reglas de confidencialidad y a la protección de información que afecte a la seguridad nacional ${ }^{63}$.

\subsubsection{Restricciones al descubrimiento de información}

En primer lugar, la regla 81 (1) señala que no están sujetos a revelación los informes, memorandos o documentos internos de trabajo preparados por las partes. Así mismo, la regla 81 (2) regula los casos en los cuales el fiscal tiene información que debe ser revelada pero cuyo descubrimiento puede perjudicar investigaciones en curso o futuras. En este caso, podrá pedir a la Sala que determine si debe ser revelada esa información o no. En todo caso, estos elementos sujetos a restricción no pueden constituirse como evidencia en juicio si antes no son descubiertos a la defensa.

Por otra parte, las reglas 81 (3) y 81 (4) regulan las situaciones donde se han tomado medidas para asegurar la confidencialidad de la información y la seguridad de víctimas y testigos ${ }^{64}$. En este caso, no se puede revelar este tipo de elementos probatorios si no se sigue el procedimiento establecido para ello en el Estatuto. Igualmente, el artículo 68 decreta que si la revelación de información puede poner en peligro la situación del testigo o su familia, el fiscal podrá optar por descubrir resúmenes

"Decision on the Final System of Disclosure and the Establishment of a Timetable", ICC-01/04-01/06. 15 May 2006, pars. 73-75.

61 En virtud de esta regla, se dice que la fiscalía debe cumplir con la obligación del descubrimiento de "manera continua", es decir, que va más allá de la etapa del juicio y se extiende a la etapa de apelaciones. Kai Ambos. Treatise on International Criminal Law, cit., p. 523.

62 Sobre lo que debe entenderse por "todo" la jurisprudencia de la CPI ha desarrollado dos interpretaciones diferentes. La primera, denominada "the bulk rule", según la cual sólo el material que será usado en la confirmación de cargos debe ser descubierto a la defensa y comunicado a la scP; la segunda, "the totality rule", conforme a la cual el fiscal debe descubrir los medios de prueba de verdadera relevancia para el caso (incriminatorios o exculpatorios) y extiende el alcance de la comunicación a la SCP de todo el material intercambiado entre las partes sin importar su intención de hacerlo valer en la confirmación de cargos. Para ver en mayor detalle, Kai Ambos. Treatise on International Criminal Law, cit., p. 527, y Alexander Heinze. Ob. cit., pp. 36, 45, 77, 238, 241, 249, 384, 412, 418, 499, 510.

63 En concordancia con las reglas 130 y 131 (2).

64 En concordancia con los artículos 54, 68, 72, y 93 del ECPI. 
de ese medio probatorio o información. Sin embargo, esta medida no puede ir en detrimento de los derechos del acusado.

Finalmente, la regla 81 (5) señala que el material o información no descubierto bajo el marco del artículo 68 (5) no puede ser plena prueba si no se revela previamente a la defensa. Lo mismo indica la regla 81 (6) para el investigado quien puede enviar un resumen de la información relevante cuando su descubrimiento pueda poner en peligro al testigo o su familia. Empero, estos mecanismos probatorios no tienen la capacidad de constituirse en evidencia si no se descubren con anterioridad al ente acusador ${ }^{65}$.

\subsubsection{Información y comunicación privilegiadas}

La regla 73 constituye una excepción al descubrimiento enmarcada en la sección I ("La prueba")66. Se refiere a la comunicación e información que hace parte de las relaciones cliente-abogado y otros grupos de personas donde sea razonable esperar que las comunicaciones sean confidenciales. En cuanto a la relación cliente-abogado establece la regla 73 (1) que esta información no está sujeta a revelación a menos que el cliente consienta por escrito en ello o que lo divulgue voluntariamente a un tercero y ese tercero lo demuestre. Igualmente, la regla 73 (2) señala que la información originada en otras relaciones de confidencialidad no estará sujeta a divulgación si la Sala decide que (1) esas comunicaciones hacen parte de una relación de confidencialidad de la cual se espera razonablemente privacidad y no divulgación; (2) la confidencialidad es indispensable para ese tipo de relación; y (3) el reconocimiento de ese privilegio promovería los objetivos del Estatuto y de las RPP.

No obstante lo anteriormente señalado, el Código de Conducta Profesional de los Abogados $^{67}$ (en adelante, CCPA) señala que la información confidencial de la relación cliente-abogado puede ser revelada cuando lo ordene la Corte.

Por otro lado, la regla 73 (4) indica que ningún documento relacionado con el trabajo del Comité Internacional de la Cruz Roja puede ser descubierto, ni siquiera

65 Finalmente, aunque las limitaciones al descubrimiento de información se verán en detalle en el siguiente capítulo, conviene decir que la Corte estableció que como regla general las declaraciones deben ser descubiertas de manera completa a la defensa, por lo que cualquier restricción en descubrimiento sobre nombres o partes, o ambos, de las declaraciones de los testigos que la Fiscalía quiere hacer valer en la confirmación de los cargos debe ser autorizada por el juez único bajo el procedimiento establecido en la regla 81 de las RPP. Ibídem, par. 101. Las salas deben justificar plenamente las autorizaciones para restringir el descubrimiento de información dando una explicación completa sobre las razones que llevan a esa determinación. Al respecto, véase también International Criminal Court. Prosecutor v. Bahar Idriss Abu Garda. "Public Redacted Version of the 'First Decision on the prosecution's Request for Redactions' issued on 14 August 2009”, ICC-02/05-02/09. 20 August 2009, par. 11.

66 Y no en la sección II (divulgación de documentos e información) del capítulo 4 de las RPP.

67 Corte Penal Internacional. Asamblea de los Estados Partes. Resolución ICC-ASP/4/Res.1, por la que se aprueba el Código de Conducta Profesional de los Abogados, 2 de diciembre de 2005, artículo 8. 
mediante testimonio de alguien que haya sido o sea su funcionario o empleado, salvo que el mismo Comité no se oponga por escrito a la revelación o haya renunciado a ese privilegio, o que esa información se encuentre en declaraciones o documentos públicos del Comité. Aquí conviene resaltar que, según la regla 73 (5), las partes pueden buscar esa información de fuente distinta si así lo desean. Por último, indica la regla 73 (6) que si esa información es de gran importancia la Corte puede contactar con el Comité con la finalidad de resolver el problema.

\subsubsection{Artículo 54 (3) (e) y regla 82}

Estos artículos deben leerse conjuntamente ${ }^{68}$ pues versan sobre el no descubrimiento de material exculpatorio reunido por la fiscalía a través de acuerdos confidenciales con una tercera parte. El artículo 54 le da al fiscal el poder para obtener documentos o información sujetos a acuerdos confidenciales ${ }^{69}$ y con el único propósito de conseguir nueva evidencia (estos convenios tienen lugar únicamente en situaciones excepcionales) ${ }^{70}$.

Ahora bien: este material, como se dijo, no tiene la virtud de ser evidencia en juicio, salvo si existe el consentimiento previo del proveedor de la información y si se ha descubierto con anterioridad a la defensa ${ }^{71}$. Si el ente persecutor presenta como prueba elementos logrados con acuerdo de confidencialidad, la sPI no puede ordenar que se presenten pruebas adicionales adquiridas de quien haya suministrado los documentos iniciales, ni convocarlo con el fin de recabar elementos materiales probatorios adicionales ${ }^{72}$. Además, si el fiscal llama a un testigo para que presente como evidencia información protegida bajo estos acuerdos, la Sala no puede ${ }^{73}$ obligarlo a responder una pregunta relacionada con el material sujeto a reserva si la persona no está dispuesta a hacerlo argumentando esa circunstancia ${ }^{74}$.

68 Brando Fiori. Ob. cit., p. 279.

69 La razón de ser de esta disposición es que la efectividad de las investigaciones del fiscal depende en gran medida de la cooperación de los Estados quienes tendrán más disposición de colaborar cuando la información que ellos proveen permanece confidencial. Brando Fiori. Ob. cit., p. 281.

70 International Criminal Court. Prosecutor v. Thomas Lubanga Dyilo. "Decision on the consequences of non-disclosure of exculpatory materials covered by article 54 (3) (e) agreements and the application to stay the prosecution of the accused, together with certain other issues raised at the status conference on 10 June 2008", ICC-01/04-01/06. 13 June 2008, par. 71.

71 CPI-RPP. Regla 81 (1).

72 CPI-RPP. Regla 82 (2).

73 CPI-RPP. Regla 82 (3).

74 En resumen, cuando la fiscalía acepta material bajo condiciones de confidencialidad, potencialmente se pone en una situación donde o no descubre material que por regla general descubriría o rompe un acuerdo de confidencialidad con el proveedor de la información. International Criminal Court. Prosecutor v. Thomas Lubanga Dyilo. "Judgement on the appeal of the prosecutor against the decision of Trial Chamber I entitled 'Decision on the consequences of non-disclosure of exculpatory materials covered by article 54 (3) (e) agreements and the application to stay the prosecution of the accused, together with other issues raised at the status conference on 10 June 2008'”, ICC-01/04-01/06, 2008, par. 43. 
Por último, la Sala tampoco está facultada para ordenar la producción de medios de prueba distintos para los cuales el consentimiento del descubrimiento se hizo. Estas disposiciones aplican para la defensa mutatis mutandis ${ }^{75}$.

\subsubsection{Remedios y sanciones en caso de vulneración al deber de descubrimiento de información}

Las normas de la CPI no establecen de manera explícita remedios o sanciones en caso de infringir el deber de descubrimiento ${ }^{76}$. Empero, pueden tenerse en cuenta tres disposiciones: (1) la regla 121 (8) en relación con el descubrimiento para la confirmación de cargos, prescribe que la Sala no toma cargos ni medios de prueba presentados extemporáneamente; (2) el artículo 69 (7) habilita a la Corte para excluir evidencia obtenida con violación de derechos humanos o del Estatuto; y (3) el artículo 71 señala que la Corte puede sancionar a personas que han actuado de mala conducta, categoría dentro de la cual se incluyen comportamientos como la interrupción de los procedimientos o las negativas a cumplir con sus mandatos ${ }^{77}$.

\section{PRINCIPIO DE PROPORCIONALIDAD Y DESCUBRIMIENTO PROBATORIO}

Con la finalidad de alcanzar un entendimiento íntegro de las instituciones que aquí convergen y a través del cual sea posible una evaluación crítica del desarrollo del principio de proporcionalidad en el disclosure del proceso penal internacional, se analizará la función del principio de proporcionalidad en el descubrimiento probatorio a través de los puntos de contacto entre estas dos figuras. Este desarrollo, como se verá en el siguiente apartado, tiene lugar en lo que aquí se denomina "proceso ponderativo".

\subsection{Siempre que se soliciten restricciones al descubrimiento probatorio debe haber aplicación del principio de proporcionalidad}

La función del principio de proporcionalidad en el marco del descubrimiento probatorio se circunscribe a las restricciones ${ }^{78}$ que a esta figura se hagan, dado que es

75 CPI-RPP. Regla 82 (5).

76 Véase en general Michele Cianello. "Disclosure before the ICC: The emergence of a new form of policies implementation system in International Criminal Justice?", en International Criminal Law Review, vol. 10, Leiden, Brill, 2010, pp. 23-42; Kai Ambos. "The First judgement of the International Criminal Court (Prosecutor v. Lubanga): A comprehensive analysis of the legal issues", en International Criminal Law Review, vol. 12, Leiden, Brill, 2012, pp. 115-153, disponible en [https:// papers.ssrn.com/sol3/papers.cfm?abstract_id=2030751] (consulta: 7 de julio de 2019); Helen Brady. Disclosure of Evidence, cit., pp. 403 y 412.

77 Véase en mayor detalle: Alexander Heinze. Ob. cit., pp. 452-477.

78 Sobre el principio de proporcionalidad como noción básica en el derecho procesal penal para determinar la constitucionalidad de las restricciones a derechos fundamentales, Jaime Bernal Cuéllar 
allí donde confluyen el deber de persecución penal y el mandato de respeto por los derechos del acusado, siendo necesario compatibilizarlos y no optar por excluir uno u otro, ya que esta salida no sería compatible con los estándares internacionales de protección de los derechos humanos ni con la filosofía misma que inspira el funcionamiento de la CPI.

\subsection{Tres "modalidades" de afectación al descubrimiento probatorio en las que debe intervenir el principio de proporcionalidad}

El derecho del sospechoso al acceso a la información con que cuenta la fiscalía para sostener su teoría del caso puede ser mermado de diferentes maneras, bien sea por la no revelación del material, por una revelación tardía o por una revelación parcial. En cualquiera de estos escenarios debe existir un pronunciamiento directo del juez señalando por qué debe restringirse el descubrimiento de medios de prueba en esas circunstancias.

Ahora bien: el principio de proporcionalidad puede verse desde otros puntos de vista en el ámbito del descubrimiento probatorio, verbigracia, la proporcionalidad entre la sanción y la conducta que infringe el deber de descubrir información o sobre lo que debe entenderse por una "facilidad adecuada" teniendo en cuenta que no existe una igualdad matemática sino proporcional frente a las facultades de la fiscalía. Sin embargo, ese no es el objetivo del presente trabajo, cuyo análisis se hace desde una perspectiva del principio de proporcionalidad como criterio para dirimir disputas entre principios.

\subsection{Principio de proporcionalidad y restricciones al descubrimiento probatorio por motivos de seguridad nacional}

El ejercicio de ponderación en estos eventos debe ser cauteloso pues las circunstancias que rodean esta excepción la hacen sumamente delicada. Por ejemplo, autorizar la divulgación de información en contra de la voluntad de un Estado puede poner en peligro las relaciones de cooperación entre la CPI y esa nación y bien se sabe que la efectividad del funcionamiento de la Corte depende, en gran medida, de la voluntad cooperativa de los Estados. Además, no puede soslayarse el hecho de que el motivo de seguridad nacional per se posee un peso abstracto bastante elevado que puede predisponer al juez en el ejercicio ponderativo.

Para finalizar, debe decirse que, en este evento, a pesar de optarse por la no divulgación, el juez está habilitado por el Estatuto para inferir, de ese material, la existencia de he$\operatorname{chos}^{79}$, lo cual pone al acusado en una desventaja manifiesta de cara al ente persecutor.

y Eduardo Montealegre Lynett. El proceso penal, tomo I: Fundamentos constitucionales y teoría general, cit., pp. 387-456. Véase también Carlos Bernal Pulido. Ob. cit., pp. 81 y ss. 


\subsection{Principio de proporcionalidad, restricciones al descubrimiento probatorio por posibles afectaciones a investigaciones en curso o futuras y las restricciones a la revelación de documentos personales de investigación}

El elemento de la temporalidad es nuclear en este escenario. El principio de proporcionalidad estará orientado a determinar si la restricción a la divulgación de información resulta idónea, necesaria y proporcional en sentido estricto en ese preciso momento en que se solicita la medida ya que, una vez concluidas las investigaciones, la subregla resultante del ejercicio ponderativo perderá su fundamento principal, la causa que justificó la limitación, ergo, el descubrimiento deviene imperativo pues no existirán ya tales pesquisas.

Para terminar, la no divulgación de documentos personales de investigación ha sido una regla importada del procedimiento penal norteamericano cuyo fundamento se encuentra en la preservación de la integridad de las investigaciones ${ }^{80}$ y la estrategia procesal de las partes. Usualmente no se relativiza esta garantía, sin embargo, nada obsta para que pueda presentarse esta situación en virtud de la función del principio de proporcionalidad.

\subsection{Principio de proporcionalidad y restricciones al descubrimiento probatorio con el objetivo de proteger la integridad de víctimas o testigos y sus familias}

El objetivo de proteger la seguridad de víctimas y testigos y sus familias es uno de los motivos más invocados para conseguir una restricción al descubrimiento. Merece especial atención el problema que surge cuando se asume que el acusado puede intimidar testigos una vez se descubra su identidad pues, como acertadamente señala el profesor Kai Ambos, se convierte la presunción de inocencia en una presunción de culpabilidad ${ }^{81}$.

\subsection{Principio de proporcionalidad y las comunicaciones e información privilegiadas}

El CCPA, como se observó, prevé la posibilidad de divulgar esta información cuando la Corte así lo ordene. Este artículo es una puerta abierta para la negación de esta garantía, lo cual es sumamente peligroso dado que no proteger esta clase de comunicaciones puede llevar a una seria violación del principio de igualdad de $\operatorname{armas}^{82}$,

80 International Criminal Court. Prosecutor v. Dominic Ongwen. "Decision Concerning the Redaction and Transmission of Documents to the Defence", ICC-02/04-01/15. 15 April 2015, par. 18.

81 Kai Ambos. Treatise on International Criminal Law, cit., p. 544.

82 Cristoph Safferling. International Criminal Procedure, cit., p. 507. 
máxime si se tiene en cuenta que las $\mathrm{RPP}^{83}$ habilitan a la Corte para desconocer ese privilegio si considera que con él no se promueven los objetivos de estas reglas o del Estatuto, una disposición bastante amplia que puede dar lugar a un elevado número de interpretaciones en el marco de una ponderación de principios.

Es importante mencionar que el desconocimiento de esta garantía con el objetivo de salvaguardar otros intereses ya ha tenido lugar en otros contextos. Por ejemplo, el Tribunal Penal Internacional para Ruanda determinó que "si la salud del acusado es la razón de una violación del derecho de otros acusados a un juicio expedito, el informe médico de este acusado pierde su privilegio" 84 .

\subsection{Principio de proporcionalidad y descubrimiento de material exculpatorio adquirido con acuerdo de confidencialidad}

Esta es sin duda una de las problemáticas más interesantes que surgen de las relaciones entre el principio de proporcionalidad y la obligación de descubrimiento probatorio por parte de la fiscalía. Por un lado, el ente acusador tiene el deber continuo de divulgar este material tan pronto como sea posible y, por otro, tiene que respetar los acuerdos de confidencialidad mediante los cuales llegó a esos elementos probatorios y que, según el propio Estatuto, no puede revelar sin el consentimiento del informante.

A primera vista, es factible que se genere un prejuicio en el juez que afecte el ejercicio ponderativo de modo tal que se incline por la declaración de inocencia, sobreponiéndola a los acuerdos de confidencialidad. Sin embargo, puede pensarse también en un caso difícil, donde la divulgación del material tenga la virtud de generar efectos devastadores en un determinado contexto: ¿debe condenarse al acusado con el objeto de evitar esa circunstancia o será necesario optar por su inocencia? Este tópico merece una investigación propia e independiente, empero, en las próximas páginas se verá si esta circunstancia ha tenido lugar en la jurisprudencia de la CPI y, de ser así, de qué manera ha sido resuelta.

\section{DESARROLLO DEL PRINCIPIO DE PROPORCIONALIDAD EN EL 'DISCLOSURE' DEL PROCESO PENAL INTERNACIONAL: EL PROCESO PONDERATIVO}

A continuación se verá el procedimiento para llegar a las mencionadas restricciones, la carga argumentativa que deben cumplir las partes y los factores que ha establecido la Corte para llevar a cabo la ponderación de los intereses encontrados. Las

83 RPP-CPI. Regla 73 (2) (c).

84 International Criminal Tribunal for Rwanda. Prosecutor v. Karemera et al., Trial Chamber III. "Decision on motion for Disclosure of Medical Information and Extension of Time", ICTR-98-44-T. 28 August 2009, par. 10 (traducción propia). 
afirmaciones consagradas en las próximas páginas aplican tanto para los eventos de la regla 81 (2) ${ }^{85}$ como para los de la regla 81 (4) de las $\operatorname{RPP}^{86}$.

\subsection{Escenario judicial en el cual se toma la decisión que autoriza el no descubrimiento de información: la audiencia 'ex parte'}

Toda restricción, como se veía anteriormente, es una excepción al deber de divulgación, por lo cual se exige autorización expresa del juez, quien puede dar lugar a ella bien sea por iniciativa propia o a solicitud de parte ${ }^{87}$. Conforme a la regla 81 (2), la Sala celebrará una vista ex parte para tratar esta cuestión, escenario en el cual, como se verá, el principio de proporcionalidad tiene un papel esencial.

Según los artículos 61 (1) y 61 (2) del Estatuto, el procesado tiene derecho a estar presente en la confirmación de cargos, prerrogativa que se extiende, en principio, a todas las fases del proceso, desde su aparición inicial a la mencionada audiencia ${ }^{88}$. Sin embargo, algunas disposiciones del Estatuto y de las RPP prevén la posibilidad de llevar a cabo procedimientos ex parte en ausencia de la defensa, concretamente la regla 81 (2) en relación con la determinación de excepciones al descubrimiento con la finalidad de no entorpecer investigaciones actuales o futuras.

Por otro lado, la regla 81 (4) señala que la Sala puede tomar las medidas necesarias para asegurar la confidencialidad de la información y la seguridad de víctimas y testigos. En ausencia de expresa prohibición, una de ellas son los procesos ex parte, específicamente previstos por el Estatuto para cuestiones de seguridad nacional bajo el artículo 72 y la protección de víctimas y testigos según el artículo 68 y la regla 88 de las $\operatorname{RPP}^{89}$.

Este modo de proceder para adoptar una decisión, como lo ha afirmado la Corte desde el caso Lubanga, es la excepción, no la regla general ${ }^{90}$ y únicamente debe permitirse $\mathrm{si}^{91}$ :

85 Ibídem, par. 97.

86 De la misma manera, se predican de aquellos casos en lo que se busca la restricción de acceso a documentos a través de niveles más estrictos de clasificación. International Criminal Court. Prosecutor v. Francis Kirimi Muthaura, Uhuru Muigai Kenyatta and Mohammed Hussein Ali. "Decision on the Confidential Redacted Version of the Article 58 Application”, ICC-01/09-02/11. 22 July 2011, par. 10.

87 RPP-CPI Regla 81 (4).

88 International Criminal Court. Prosecutor v. Thomas Lubanga Dyilo. "Decision Establishing General Principles Governing Applications to Restrict Disclosure pursuant to Rule 81 (2) and (4) of the Rules of Procedure and Evidence", ICC-01/04-01/06. 19 May 2006, par. 9.

89 Ibídem, par. 10.

90 Ibídem, par. 12.

91 Ibídem, par. 13. 
(1) sirve para el alcance de un objetivo suficientemente importante;

(2) es necesario en el sentido de que ninguna menor medida podría ser suficiente para alcanzar un resultado similar; $y$

(3) el perjuicio al interés de la defensa de desempeñar un rol más activo en los procedimientos debe ser proporcional al beneficio que se deriva de tal medida.

La noción de ex parte, como se expresa en la norma 24 (4) del Reglamento de la Secretaría ${ }^{92}$, puede tener dos significados. El primero se refiere a procedimientos donde la fiscalía, la defensa o cualquier otro participante (o combinaciones entre estos), si bien saben que esos procedimientos existen, no tienen la oportunidad de ser escuchados o de presentar documentos, materiales u órdenes; y el segundo, conforme al cual son procedimientos cuya existencia desconocen la fiscalía, la defensa o cualquier otro participante ${ }^{93}$.

En virtud de su naturaleza, se considera que la defensa no tiene derecho a recibir ninguna versión redactada de ningún documento archivado como ex parte o de las transcripciones de cualquier audiencia celebrada bajo esta modalidad de conformidad con las reglas 81 (2) y 81 (4) porque el significado mismo de ex parte excluye cualquier oportunidad para el procesado de presentar argumentos, documentos, materiales u órdenes en relación con el contenido específico de la solicitud de la fiscalía ${ }^{94}$.

Para terminar, es oportuno señalar que, bajo los mismos principios, la defensa también puede aplicar, siguiendo la regla 81 (4) de las RPP, a esta clase de procedimientos requiriendo la ausencia del ente acusador ${ }^{95}$.

\subsection{Metodología de la Sala en la audiencia ex parte: "two-step approach"}

En la vista ex parte lo primero que debe hacer el juez es determinar si se trata de información descubrible y, en caso afirmativo, entra posteriormente al estudio de la solicitud para determinar si hay lugar o no a las restricciones. Esta forma de proceder es lo que la jurisprudencia de la CPI ha denominado "two-step approach"96.

92 Corte Penal Internacional. Presidencia de la Corte Penal Internacional. Documentos oficiales de la Corte Penal Internacional. ICC-BD/03-01-06. Reglamento de la Secretaría, 6 de marzo de 2006.

93 International Criminal Court. Prosecutor v. Thomas Lubanga Dyilo. "Decision Establishing General Principles Governing Applications to Restrict Disclosure pursuant to Rule 81 (2) and (4) of the Rules of Procedure and Evidence", ICC-01/04-01/06. 19 May 2006, par. 14.

94 Ibídem, par. 18.

95 Ibídem, par. 20.

96 International Criminal Court. Prosecutor v. William Samoei Ruto and Joshua Arap Sang. "Decision 
Es en este segundo paso donde el principio de proporcionalidad tiene su función más importante pues se requiere encontrar un balance entre el interés de la protección de la vida y la integridad de las víctimas y los testigos o sus familiares, la garantía de confidencialidad de información o la efectividad de las investigaciones y los derechos del acusado.

La doctrina de la CPI, cuyo desarrollo se verá en el siguiente apartado, proporciona tres principios generales en caso de no descubrimiento, a saber: (1) principio de necesidad: las medidas de protección sólo deben ser garantizadas después de haber agotado la posibilidad de emplear medidas menos extremas; (2) principio de proporcionalidad: los medios que se adopten deben ser estrictamente limitados a las exigencias de la situación; y (3) las medidas no deben vulnerar el derecho del procesado a un juicio justo e imparcial ${ }^{97}$.

En este contexto, conviene resaltar que las medidas mediante las cuales se ha cristalizado la restricción al deber de divulgación son de distinta índole y, como se había anticipado en el estudio de las relaciones entre descubrimiento probatorio y principio de proporcionalidad, pueden versar sobre un descubrimiento parcial o un descubrimiento tardío. No hay lugar a la no revelación absoluta en el sentido de que pueda hacerse valer como prueba un elemento que jamás fue descubierto pues, como se vio, la normativa de la CPI no permite la inclusión de medios probatorios que no sean revelados de manera previa y adecuada. Lo que puede ocurrir es que en un determinado momento no se descubra la totalidad de un medio de prueba pero que con posterioridad se proceda a su divulgación, por lo que este evento quedaría comprendido dentro de la categoría del descubrimiento tardío. Específicamente y conforme a la práctica de la CPI, estas medidas han sido las "redacciones" de documentos ${ }^{98}$ y las autorizaciones para delayed disclosure ${ }^{99}$.

En relación con las redacciones, debe decirse que el principio general que las gobierna es la necesidad de prevenir la identificación de los solicitantes. Las redacciones consisten en el cubrimiento o alteración de cierta información en los documentos,

on Disclosure of Information related to Prosecution Intermediaries”, ICC-01/09-01/11. 4 September 2013, par. 26

97 International Criminal Court. Prosecutor v. Thomas Lubanga Dyilo. "Judgment on the Appeal of Mr. Thomas Lubanga Dyilo against the Decision of Pre-Trial Chamber I entitled 'First Decision on the Prosecution Requests and Amended Requests for Redactions under Rule 81',", ICC-01/04-01/06-773. 14 December 2006, pars. 21 and 33.

98 A modo de ejemplo, puede consultarse International Criminal Court. Prosecutor v. William Samoei Ruto and Joshua Arap Sang. "Decision on Prosecution Application to Redact Investigator's Identifying Information and to Vary the Redaction Protocol”, ICC-01/09-01/11. 21 December 2012.

99 A modo de ejemplo, puede consultarse International Criminal Court. Prosecutor v. William Samoei Ruto and Joshua Arap Sang. "Confidential Redacted Version of 'Decision on First Prosecution Application for Delayed Disclosure of Witness Identities””, ICC-01/09-01/11. 4 January 2013. 
específicamente cualquier dato sobre la identidad de los aplicantes ${ }^{100}$. Sin embargo, como se verá más adelante, las redacciones también se han utilizado para garantizar la confidencialidad de información y la posibilidad de adelantar investigaciones en curso o futuras, verbigracia, mediante la redacción del lugar donde se llevan a cabo entrevistas.

Por otra parte, en relación con las autorizaciones para delayed disclosure, es imperativo señalar que los límites de tiempo para cumplir con las obligaciones en materia de descubrimiento probatorio establecidos en la normativa de la CPI garantizan ${ }^{101}$ el derecho a recibir de manera oportuna la información para la preparación del caso. Así mismo, es necesario tener en cuenta que a pesar de que el Estatuto ${ }^{102}$ señala que el descubrimiento debe tener lugar con suficiente antelación al comienzo del juicio, en ocasiones se ha ordenado el descubrimiento con posterioridad al inicio de este ${ }^{103}$.

No obstante lo antes señalado, esos límites son indicativos de los plazos mínimos ${ }^{104}$ y el juez puede establecer los tiempos de divulgación que él considere adecuados para el caso concreto. Establecidos los términos finales, cualquier demora más allá de estos debe ser autorizada sólo en circunstancias excepcionales ${ }^{105}$, caso en el cual

100 Incluyendo: el nombre, nombre de los padres, lugar de nacimiento, fecha de nacimiento, tribu o grupo étnico, ocupación, estatus civil, número de dependientes, tipo de prueba de identidad proporcionada, dirección de residencia actual y lugar de origen (pero no el país), número de teléfono y correo electrónico, idiomas que habla, nombre de las víctimas o testigos del mismo incidente, características específicas de la lesión, pérdida o daño supuestamente sufridos, nombre y datos del intermediario que ayuda a la víctima a presentar la solicitud. International Criminal Court. Prosecutor v. Dominic Ongwen. "Decision on Legal Representation, Appointment of Counsel for the Defence, Protective Measures and Time-limit for Submission of Observations on Applications a/0010/06, a/0064/06, to a/0070/06, a/0081/06 to a/0104/06 and a/0111/06 to a/0127/06", ICC-02/04-01/05. 1 February 2007, par. 21.

101 International Criminal Court. Prosecutor v. Callixte Mbarushimana. "Decision on Issues Relating to Disclosure", ICC-01/04-01/10. 30 March 2011, par. 20.

102 ECPI, artículo 64 (3) (e).

103 International Criminal Court. Prosecutor v. William Samoei Ruto and Joshua Arap Sang. "Confidential Redacted Version of 'Decision on First Prosecution Application for Delayed Disclosure of Witness Identities"”, ICC-01/09-01/11, 4 January 2013, par. 29. Tambien ver International Criminal Court. Prosecutor v. William Samoei Ruto and Joshua Arap Sang. "Confidential Redacted Version of the 'Decision on the Prosecution's Renewed Request for Delayed Disclosure of Identity of Witness 534'”, ICC-01/09-011. 2 May 2013, par. 27-33.

104 International Criminal Court. Prosecutor v. Dominic Ongwen. "Decision Setting the Regime for Evidence Disclosure and Other Related Matters", ICC-02/04-01/15. 27 February 2015, par. 17. Véase también International Criminal Court. Prosecutor v. William Samoei Ruto and Joshua Arap Sang. "Decision Setting the Regime for Evidence Disclosure and Other Related Matters", ICC-01/09-01/11. 6 April 2011, par. 10.

105 International Criminal Court. Prosecutor v. William Samoei Ruto and Joshua Arap Sang. "Confidential Redacted Version of 'Decision on First Prosecution Application for Delayed Disclosure of Witness Identities"”, ICC-01/09-01/11. 4 January 2013, par. 26. 
será obligatorio cumplir con los requerimientos del principio de proporcionalidad ${ }^{106}$ de la misma manera en que se demanda para la autorización de redacciones ${ }^{107}$.

Finalmente, es necesario señalar que, hasta el momento, no han existido en las decisiones de la CPI ejercicios de proporcionalidad para determinar si se flexibiliza la facultad de no descubrir documentos internos de trabajo, información obtenida con acuerdo de confidencialidad o información privilegiada. En estos casos el debate ha girado en torno a si los documentos constituyen elementos de trabajo interno ${ }^{108}$, si existe o no consentimiento para la divulgación de información obtenida con acuerdo de confidencialidad ${ }^{109}$ o si se trata o no de información privilegiada ${ }^{110}$. En otras palabras, la Corte ha protegido el derecho a no revelar esta información incluso si estos intereses entran en conflicto con otros igualmente exigibles. Sin embargo, cuando este tipo de material se quiere descubrir, pero con algún tipo de restricción, verbigracia, redacciones, debe la Sala proceder al estudio ${ }^{111}$ correspondiente para determinar si esas limitaciones son compatibles ${ }^{112}$ con los derechos del procesado ${ }^{113}$.

\subsection{Desarrollo del proceso ponderativo}

La autorización para el no descubrimiento requiere una evaluación cuidadosa donde se tienen en cuenta varios intereses en juego ${ }^{114}$. Estas medidas se pueden tomar de

106 International Criminal Court. Prosecutor v. Francis Kirimi Muthaura and Uhuru Muigai Kenyatta. "Decision on the Prosecution's Application for Relief pursuant to Decision 451, Rule 81 (2) and Regulation 35, 9 January 2013, ICC-01/09-02/11-595-Conf”, ICC-01/09-02/11, 2017, pars. 5-10.

107 International Criminal Court. Prosecutor v. William Samoei Ruto and Joshua Arap Sang. "Confidential Redacted Version of 'Decision on First Prosecution Application for Delayed Disclosure of Witness Identities””, ICC-01/09-01/11. 4 January 2013, pars. 27-31.

108 Sin embargo, si se quieren hacer valer como prueba, pero con redacciones, deben cumplirse los requisitos exigidos por el principio de proporcionalidad. International Criminal Court. Prosecutor $v$. Dominic Ongwen. "Decision Concerning the Redaction and Transmission of Documents to the Defence", ICC-02/04-01/15. 15 April 2015, par. 18

109 International Criminal Court. Prosecutor v. Dominic Ongwen. "Decision on the Prosecution's Application under Article 53 (3) (f) to Apply Redactions to Documents Obtained under Article 54 (3) (e)”, ICC-02/04-01/15. 2 March 2016, par. 4.

110 International Criminal Court. Prosecutor v. Callixte Mbarushimana. "Decision on the Review of Potentially Privileged Material", ICC-01/04-01/10. 15 June 2011.

111 No obstante, este estudio no se llevó a cabo en el caso Mbarushimana, donde se entendió que el descubrimiento en versión redactada era por sí sólo suficiente para resolver la tensión entre la confidencialidad de la información y el derecho del sospechoso al juicio justo. International Criminal Court. Prosecutor v. Callixte Mbarushimana. "Decision on Prosecution's Application for Authorisation to Disclose a Document received pursuant Article 54 (3) (e) in redacted form”, ICC-01/04-01/10, 2011.

112 Ídem.

113 International Criminal Court. Prosecutor v. Dominic Ongwen. "Decision Concerning the Redaction and Transmission of Documents to the Defence", ICC-02/04-01/15. 15 April 2015, par. 19.

114 International Criminal Court. Prosecutor v. Germain Katanga. "Judgment on the Appeal of the Prosecutor against the Decision of Pre-Trial Chamber I entitled 'First Decision on the Prosecution Request for Authorisation to Redact Witness Statements"”, ICC-01/04-01/07 (OA), 2008, par. 66. 
oficio por el juez o a solicitud de parte, soportándose siempre en un razonamiento suficiente ${ }^{115}$, donde deberán proporcionarse argumentos de tal calibre que sean capaces para convencer a la Sala de tomar dicha decisión. Es por lo anterior por lo que se exige a quien las solicita que desarrolle de manera detallada para cada solicitud el cumplimiento de los siguientes criterios $^{116}$ :

(1) señalar la base legal para cada redacción;

(2) explicar y analizar cada hecho individual que justifique la medida de protección que se propone;

(3) evaluar adecuadamente la viabilidad y adecuación de todas las medidas de protección disponibles;

(4) establecer que la no divulgación es la única medida apropiada; y

(5) demostrar que la medida se limita a lo estrictamente necesario ${ }^{117}$ en relación con los derechos del sospechoso.

En ese orden de ideas la Sala de Apelaciones (en adelante, SA) estableció los factores que debe abordar el juez para proceder a la excepción del deber de divulgación, los cuales pueden resumirse en ${ }^{118}$ :

(1) la necesidad de la medida de protección;

(2) debe ser la medida necesaria menos intrusiva para proteger los derechos de la persona en cuestión, la confidencialidad de la información o la preservación de la facultad de adelantar investigaciones actuales o futuras; y

(3) desde una evaluación general ${ }^{119}$, la medida de protección no puede ser perjudicial o inconsistente con los derechos del acusado a un juicio justo e imparcial.

115 International Criminal Court. Prosecutor v. Jean-Pierre Bemba Gombo. "Public redacted version of ICC-01/05-01/08-48-US-Exp Decision Concerning the Prosecutor's Proposals for Redactions", ICC-01/05-01/08. 23 July 2008, par. 26.

116 Ibídem, par. 40.

117 International Criminal Court. Prosecutor v. Dominic Ongwen. "Decision on Legal Representation, Appointment of Counsel for the Defence, Protective Measures and Time-limit for Submission of Observations on Applications a/0010/06, a/0064/06, to a/0070/06, a/0081/06 to a/0104/06 and a/0111/06 to a/0127/06", ICC-02/04-01/05. 1 February 2007, par. 23.

118 International Criminal Court. Prosecutor v. Thomas Lubanga Dyilo. "Judgment on the Appeal of Mr Thomas Lubanga Dyilo against the Decision of Pre-Trial Chamber I entitled 'First Decision on the Prosecution Requests and Amended Requests for Redactions under Rule 81'”, ICC-01/0401/06-773 (OA5). 14 December 2006, pars. 21 and 33-34.

119 International Criminal Court. Prosecutor v. William Samoei Ruto and Joshua Arap Sang. "Decision on the Prosecution's Application for Authorisation to Maintain Certain Redactions", ICC-01/09-01/11. 23 April 2013, par. 26. 
En este estudio, la Sala debe evaluar con cuidado la información que no se quiere descubrir ${ }^{120}$. Además, el juez, con una visión general del proceso in toto, tendrá en cuenta los hechos y circunstancias individuales de cada caso y de cada solicitud concreta $^{121}$.

De manera más específica, otra sala señaló que los requerimientos para autorizar la excepción al descubrimiento son ${ }^{122}$ :

(1) la existencia de un riesgo objetivamente justificable a la seguridad de la persona a la cual se quiere proteger o un riesgo de perjudicar investigaciones actuales o futuras o el carácter confidencial de la información, riesgo que debe surgir del descubrimiento particular al acusado;

(2) la infactibilidad o insuficiencia de medidas de protección menos restrictivas;

(3) una evaluación sobre si las medidas que se buscan son perjudiciales o inconsistentes con los derechos del acusado a un juicio justo e imparcial y la obligación de revisar periódicamente la decisión que las autoriza en caso de que cambien las circunstancias que les dieron lugar.

El estudio de cada uno de estos requerimientos se verá en detalle de manera inmediata.

\subsubsection{La existencia de un riesgo objetivamente justificable}

La contingencia de daño que exige la jurisprudencia de la CPI debe reunir los siguientes requisitos ${ }^{123}$ :

(1) El alegado peligro debe envolver un riesgo objetivamente justificable a la seguridad de la persona o de las investigaciones en curso o futuras o del carácter confidencial de la información en cuestión; y

(2) El riesgo debe surgir concretamente de revelar la información a la defensa y no de descubrirla al público en general. La Sala debe considerar, entre otras cosas, si el peligro se puede superar haciendo que la información sea disponible

120 International Criminal Court. Prosecutor v. Germain Katanga. "Judgment on the Appeal of the Prosecutor Against the Decision of Pre-Trial Chamber I entitled 'First Decision on the Prosecution Request for Authorisation to Redact Witness Statements"”, ICC-01/04-01/07 (OA). 13 May 2008, par. 69.

121 Ídem.

122 International Criminal Court. Prosecutor v. Francis Kirimi Muthaura and Uhuru Muigai Kenyatta. "Decision on the Protocol Establishing a Redaction Regime", ICC-01/09-02/11. 27 September 2012, par. 11 .

123 Ibídem, par. 71. 
únicamente inter-partes. En esta evaluación deben tenerse en cuenta las circunstancias particulares del procesado para determinar si existe o no posibilidad de que él o ella transmitan esa información a terceros o si de alguna manera con sus acciones pueden poner en peligro a otros.

Ahora bien, conforme a la práctica de la Corte en la evaluación del riesgo, específicamente en aquellos casos donde se ha querido proteger la seguridad de víctimas o testigos, se constata que las salas han otorgado especial peso a:

(1) las circunstancias personales del individuo que se quiere proteger;

(2) si esa persona se beneficia de otras medidas de protección;

(3) la situación de seguridad donde la persona reside ${ }^{124}$;

(4) si la persona ha recibido amenazas del procesado o sus partidarios;

(5) si la persona por sí misma ha tomado acciones para poner en peligro su propia seguridad personal ${ }^{125}$,

(6) la existencia de amenazas a los testigos debido a que están involucrados con las actividades de la Corte; $y$

(7) si las víctimas o los testigos han consentido o no en el descubrimiento de su identidad $^{126}$.

Para terminar, la CPI ha sido enfática en señalar que estas medidas tienen la finalidad de evitar la concreción del riesgo que versa sobre la seguridad de la persona o el carácter confidencial de la información que se pretende proteger o la preservación de la posibilidad de llevar a cabo investigaciones actuales o futuras y no pueden consistir en la necesidad de proteger otro tipo de intereses, verbigracia, la intimidad de los testigos, pues no existe un fundamento legal para la salvaguarda de otros derechos por esta vía ${ }^{127}$.

124 International Criminal Court. Prosecutor v. Callixte Mbarushimana. "Decision on the Prosecution's Application for Redactions pursuant to Rule 81 (2) and Rule 81 (4)", ICC-01/04-01/10. 20 May 2011, par. 13 .

125 International Criminal Court. Prosecutor v. Jean Pierre-Bemba Gombo. "Public redacted version of ICC-01/05-01/08-215-Conf Third Decision on the Prosecutor's Requests for Redactions and Related Request for the Regulation of Contacts of Jean-Pierre Bemba Gombo, ICC-01/05-01/08. 6 November 2008, par. 51.

126 International Criminal Court. Prosecutor v. Ahmad Al Faqi Al Mahdi. "Decision on the Prosecutor's Request for Redactions", ICC-01/12-01/15. 8 December 2015, par. 6.

127 International Criminal Court. Prosecutor v. Dominic Ongwen. "Decision on the Prosecutor's Request for Authorisation of non-standard Redactions, ICC-02/04-01/15. 23 December 2015, pars. 3 and 4. 


\subsubsection{La infactibilidad o insuficiencia de medidas de protección menos restrictivas}

Una vez se concluye que el riesgo existe, lo que debe hacer el juez posteriormente es confirmar si las medidas que se buscan pueden o no hacer que se supere o se reduzca ese riesgo. En caso negativo, la Sala no debe proceder a las medidas, y en caso afirmativo, los siguientes factores deben ser considerados para determinar si lo derechos del sospechoso serán restringidos únicamente en lo estrictamente necesario ${ }^{128}$ :

(1) considerar si una medida alternativa está disponible y es factible en esas circunstancias;

(2) tener en cuenta que la no divulgación se solicita en una etapa del proceso en relación con la confirmación de cargos;

(3) que la Sala evalúe cuidadosamente la relevancia de la información para la defensa; $y$

(4) analizar si el no descubrimiento resultaría en una confirmación de cargos que, vista como un todo, es injusta para el sospechoso, caso en el cual las medidas no deben ser autorizadas ${ }^{129}$.

Ahora bien: con el objetivo de plantear un escenario más garantista en relación con los derechos del acusado conviene preguntarse qué medidas alternativas al no descubrimiento podrían salvaguardar los intereses que se pretenden proteger.

La SCP en el caso Katanga estableció como ejemplo de medidas menos intrusivas que las redacciones, con las cuales se buscaba eludir la identificación de los investigadores de la fiscalía, las siguientes: evitar tomar declaraciones en pueblos pequeños o ciudades; asegurarse de que tales personas no se destaquen fácilmente de la población local; y rotar a tales investigadores una vez haya indicios de que su identificación con la Corte puede poner en peligro su seguridad o la investigación del ente persecutor ${ }^{130}$.

Igualmente, en el caso Bemba la Sala señaló que las redacciones eran muy extensas y perjudicarían los derechos de la defensa si se garantizaran, pero al unísono descubrir

128 International Criminal Court. Prosecutor v. Germain Katanga. "Judgment on the Appeal of the Prosecutor Against the Decision of Pre-Trial Chamber I entitled 'First Decision on the Prosecution Request for Authorisation to Redact Witness Statements"', ICC-01/04-01/07 (OA), 2008, par. 72.

129 Ídem.

130 International Criminal Court. Prosecutor v. Germain Katanga. "Judgment on the Appeal of the Prosecutor Against the Decision of Pre-Trial Chamber I entitled 'First Decision on the Prosecution Request for Authorisation to Redact Witness Statements"”, ICC-01/04-01/07 (OA). 13 May 2008, par. 77. 
la información habría hecho que el procesado identificara fácilmente a los testigos, por lo que la Sala encontró como única alternativa disponible el uso de resúmenes ${ }^{131}$ de las declaraciones de estos ${ }^{132}$.

Para concluir, si la Sala determina que no existe medida menos lesiva que la restricción al descubrimiento con el objetivo de salvaguardar los intereses que juegan en contra de los derechos del procesado, se procede, como se verá a continuación, a determinar si las medidas vulneran o no su derecho al fair trial.

\subsubsection{Evaluación acerca de la compatibilidad de las medidas con los derechos del acusado a un juicio justo e imparcial y la obligación de revisar periódicamente la decisión que autoriza el no descubrimiento de información}

Habiendo superado los dos primeros filtros, el juez debe someter a consideración si las medidas que se solicitan vulneran o no el derecho del procesado al fair trial, para lo cual la jurisprudencia de la Corte ha señalado que los siguientes factores deben ser evaluados ${ }^{133}$ :

(1) Al sopesar los diferentes intereses, la Sala debe garantizar que adecuadas salvaguardas tienen lugar de forma tal que sea factible proteger los intereses del sospechoso y cumplir, en la medida de lo posible, con los requerimientos de la igualdad de armas y el carácter adversarial del proceso. En caso de que sea posible una afectación del derecho del sospechoso al juicio justo, deberán tomarse medidas de contrabalanceo tales como el uso de seudónimos o códigos de letras ${ }^{134}$ en las redacciones de los documentos de que se trate o resúmenes de aseveraciones redactadas de declaraciones de testigos ${ }^{135}$. Estas medidas

131 Ídem.

132 Es importante destacar que el uso de resúmenes está autorizado por la normativa de la cPI y no se requiere un permiso especial del juez para proceder a ellos, salvo si se quieren hacer redacciones a los mismos, en cuyo caso deberá cumplirse con los requisitos propios de las restricciones a la divulgación de información. International Criminal Court. Prosecutor v. Jean-Pierre Bemba Gombo. "Public redacted version of ICC-01/05-01/08-135-Conf Second Decision on the Prosecutor's Request for Redactions", ICC-01/05-01/08. 1 October 2008, par. 57.

133 Ibídem. par. 73.

134 International Criminal Court. Prosecutor v. Francis Kimiri Muthaura and Uhuru Muigai Kenyatta. "Decision on the Prosecution's First Request for The Authorisation of Redactions, 13 December 2012, ICC-01/09-02/11-569-Conf", ICC-01/09-02/11. 3 February 2017, par. 30; International Criminal Court. Prosecutor v. William Samoei Ruto and Joshua Arap Sang. "Decision on Prosecution Applications to Redact Investigators' Identifying information and to vary the Redaction Protocol", ICC-01/09-01/11. 21 December 2012, par. 23.

135 International Criminal Court. Prosecutor v. Francis Kimiri Muthaura and Uhuru Muigai Kenyatta. "Decision on Second Prosecution Application for Delayed Disclosure of Witness Identities, 8 January 2013, ICC-01/09-02/11-593-Conf-Exp", ICC-01/09-02/11. 3 February 2017, par. 44. 
son importantes en caso de no descubrimiento de información conseguida mediante acuerdo de confidencialidad ya que en la audiencia ex parte la Sala deberá determinar si el material debería descubrirse si no existiera tal acuerdo. En caso afirmativo, el fiscal deberá buscar el consentimiento del proveedor de la información y, de no conseguirse, la Sala, ya que tiene prohibido ordenar el descubrimiento, determinará si hay lugar o no a medidas de contrabalanceo y, de ser así, señalará cuáles de ellas pueden adoptarse para garantizar el derecho del procesado a un juicio justo a pesar de la falta de divulgación ${ }^{136}$;

(2) Antes de decidir sobre la autorización de las medidas, la Sala debe dar a la defensa la mayor oportunidad posible para presentar propuestas sobre los temas involucrados sin necesidad de revelar la información que se pretende proteger; $y$

(3) Aun cuando se autorice la no revelación, esa decisión debe permanecer bajo revisión de la Sala ya que posteriormente puede ser necesario que sea descubierta bien sea en virtud de algún cambio de circunstancias o porque las razones que las justifican desaparecen ${ }^{137}$. Aquí, es importante resaltar que el fiscal debe asistir a la Sala en esta cuestión atrayendo su atención sobre ciertos factores que pueden hacer que reconsidere su decisión sobre la no divulgación.

Ahora bien: la práctica de la Corte ha mostrado que cuando la información que se pretende proteger no está relacionada directamente con el caso, o no es relevante ${ }^{138}$, la excepción al deber de descubrir medios probatorios no vulnera los derechos del sospechoso ${ }^{139}$. Así mismo, otra de las razones que se han aducido con frecuencia por parte de la CPI para afirmar que los derechos del acusado no se ven afectados es el

136 International Criminal Court. Prosecutor v. Abdallah Banda Abakaer Nourain and Saleh Mohammed Jerbo Jamus. "Decision on Article 54 (3) (e) documents", ICC-02/05-03/09. 23 November 2011, par. 16. Véase también International Criminal Court. Prosecutor v. Abdallah Banda Abakaer Nourain and Saleh Mohammed Jerbo Jamus. "Decision on the Defence Request for a Temporary Stay of Proceedings", ICC-02/05-03/09. 26 October 2012, par. 148.

137 International Criminal Court. Prosecutorv. Al Hassan Ag Abdoul Aziz Ag Mohammed Ag Mahmoud. "Decision on the Evidence Disclosure Protocol and Other Related Matters", ICC-01/12-01/18. 16 May 2018, par. 34. Véase también International Criminal Court. Prosecutor v. Ahmad Al Faqi Al Mahdi. "Decision on Issues Related to Disclosure and Exceptions thereto", ICC-01/12-01/15. 30 September 2015, par. 8 .

138 International Criminal Court. Prosecutor v. Jean-Pierre Bemba Gombo, Aimé Kilolo Musamba, Jean-Jacques Mangenda Kabongo, Fidelé Babala Wandu and Narcisse Arido. "Decision on the 'Prosecution's Application for Redactions pursuant to Rules 81 (2) and 81 (4) of the Rules of Procedure and Evidence'. Dated 6 June 2014”, ICC-01/05-01/13. 17 June 2014, par. 5.

139 International Criminal Court. Prosecutor v. William Samoei Ruto and Joshua Arap Sang. "Decision on the Prosecution's Application for Authorisation to Maintain Certain Redactions", ICC-01/0901/11. 23 April 2013, par. 40. 
hecho de que con posterioridad tendrá acceso ${ }^{140}$ a la evidencia relevante del caso y podrá ser efectivamente controvertida ${ }^{141}$. Lo mismo ha considerado el alto tribunal cuando no se revela información sobre la identidad de los miembros de la familia de los testigos o víctimas ya que ninguno de ellos es, en estricto sentido, un testigo o, de alguna manera, recurso para el fiscal ${ }^{142}$.

Para terminar, es imperativo manifestar que si bien las redacciones son admisibles, proceder a un número significativo de estas plantea problemas graves con el derecho de la defensa al fair trial dado el impacto cumulativo y la dificultad ${ }^{143}$ que implica controvertirlas $^{144}$.

\subsection{Ejemplos de excepciones al deber de descubrimiento probatorio}

Con el objeto de lograr un panorama más completo sobre la doctrina del principio de proporcionalidad en el disclosure del proceso penal ante la CPI es oportuno señalar algunos ejemplos, a cuya lectura se remite si se quiere ahondar en ellos, en los cuales se procedió al estudio concreto de los requerimientos elaborados por las salas en la práctica judicial.

Un primer ejemplo puede encontrarse en el caso Ruto and Sang, en el cual se consideró que descubrir las identidades de los investigadores podría atraer vigilancia sobre sus actividades, elevando también potenciales riesgos a la seguridad de los testigos que residían en Kenia. Esta medida se consideró necesaria, pues minimizaba las posibilidades de que se estableciera un vínculo entre los investigadores y aquellos

140 International Criminal Court. Prosecutor v. Germain Katanga and Mathieu Ngudjolo Chui. "Sixth Decision on the Prosecution Request for Authorisation to Redact the Interviews Transcripts of Witness 238", ICC-01/04-01/07. 21 April 2008, par. 12.

141 International Criminal Court. Prosecutor v. Jean-Pierre Bemba Gombo. "Public redacted version of ICC-01/05-01/08-135-Conf Second Decision on the Prosecutor's Request for Redactions", ICC01/05-01/08. 1 October 2008, par. 65.

142 International Criminal Court. Prosecutor v. Callixte Mbarushimana. "Decision on the Prosecution's Application for Redactions pursuant to Rule 81 (2) and Rule 81 (4)", ICC-01/04-01/10. 20 May 2011, par. 29

143 Este fue el caso en International Criminal Court. Prosecutor v. Jean-Pierre Bemba Gombo. "Public redacted version of ICC-01/05-01/08-48-US-Exp Decision Concerning the Prosecutor's Proposals for Redactions", ICC-01/05-01/08. 23 July 2008, par. 34.

144 Además, debe recordarse que la decisión que toma el juez no puede fundamentarse principal o exclusivamente en testigos anónimos. También conviene decir que las salas han otorgado a este tipo de testigos menor valor probatorio. International Criminal Court. Prosecutor v. Al Hassan Ag Abdoul Aziz Ag Mohammed Ag Mahmoud. "Decision on the Prosecution Motion for Authorization to File an Anonymous Summary Concerning Witness MLI-OTP-P-P0113”, ICC-01/12-01/18. 27 September 2018, par. 36 
con quienes se reunían ${ }^{145}$. Además de la inexistencia de medidas alternativas, las redacciones no vulneraban los derechos del acusado dado que el ocultamiento de la identidad de los investigadores no obstaculizaba su facultad para evaluar los documentos por ellos proporcionados y, en todo caso, el potencial perjuicio que pudiera padecer el acusado podría ser mitigado con el uso de pseudónimos ${ }^{146}$.

En este mismo caso, aunque desde otra perspectiva, se autorizó el descubrimiento tardío de la identidad de un testigo ${ }^{147}$ debido a la existencia de un riesgo objetivamente justificable soportado en el reporte de la Unidad de Víctimas y Testigos. La medida era absolutamente idónea para salvaguardar la seguridad de la persona, empero, existían otras vías de protección igualmente viables, pero que estaban en trámite, por lo cual se autorizó retrasar la revelación de manera temporal mientras estas se materializaban. Así, se encontró un balance con los derechos del acusado, a quien además se le garantizó, como medio de contrabalanceo, un resumen proporcionado por el fiscal de cualquier afirmación sustancial hecha por el testigo en su declaración ${ }^{148}$.

Para concluir, en el caso Gaddafi se retuvo la identidad de los investigadores pues haría que estos fueran rastreados al lugar donde adelantaban las investigaciones, lo cual significaría un perjuicio para futuras pesquisas. Tampoco se reveló el lugar en donde se encontraban, pues, teniendo en cuenta que los miembros del ente persecutor tenían espacios muy limitados donde podían desenvolverse, este descubrimiento podría perjudicar en el futuro la posibilidad de dirigirse a esos sitios para continuar sus labores. Entre otras cosas, se sostuvo que publicar la ubicación podría hacer que los testigos fueran identificados, poniéndolos en un riesgo injustificable ${ }^{149}$.

145 International Criminal Court. Prosecutor v. William Samoei Ruto and Joshua Arap Sang. "Decision on Prosecution Applications to Redact Investigators' Identifying Information and to Vary the Redaction Protocol", ICC-01/09-01/11. 21 December 2012, par. 21. Tambien ver International Criminal Court. Prosecutor v. Jean-Pierre Bemba Gombo, Aimé Kilolo Musamba, Jean-Jacques Mangenda Kabongo, Fidelé Babala Wandu and Narcisse Arido. "Decision on the 'Prosecution's Application for Redactions pursuant to Rules 81 (2) and 81 (4) of the Rules of Procedure and Evidence'. Dated 6 June 2014", ICC-01/05-01/13. 17 June 2014, para 9. Tambien ver International Criminal Court. Prosecutorv. Uhuru Muigai Kenyatta. "Decision on the Prosecution's First Request for Authorisation of Redactions, 13 December 2012, ICC-01/09-02/11-569-Conf"”, ICC-01/09-02/11 . 3 February 2017.

146 International Criminal Court. Prosecutor v. William Samoei Ruto and Joshua Arap Sang. "Decision on Prosecution Applications to Redact Investigators' Identifying Information and to Vary the Redaction Protocol", ICC-01/09-01/11. 21 December 2012, par. 23.

147 International Criminal Court. Prosecutor v. William Samoei Ruto and Joshua Arap Sang. "Confidential Redacted Version of 'Decision on First Prosecution Application for Delayed Disclosure of Witness Identities"”, ICC-01/09-01/11. 4 January 2013, pars. 43-48.

148 International Criminal Court. Prosecutor v. William Samoei Ruto and Joshua Arap Sang. "Confidential Redacted Version of 'Decision on First Prosecution Application for Delayed Disclosure of Witness Identities"”, ICC-01/09-01/11. 4 January 2013, par. 48.

149 International Criminal Court. Prosecutor v. Saif Al-Islam Gaddafi and Abdullah Al-Senussi. "Decision on the Prosecution's Request for Redactions pursuant to Rule 81 (2)", ICC-01/11-01/11. 16 August 2013, pars. 6-8. 
La Sala en este caso no encontró una medida menos restrictiva viable para ese momento y señaló que, dado que las redacciones sobre la identidad de los investigadores constituían discreta y limitada información de poco valor para el ejercicio de los derechos de la defensa, no existía perjuicio para esta. Así mismo, en relación con la no divulgación del sitio donde se llevaban las investigaciones, indicó que tampoco era inconsistente con el juicio justo del acusado pues se refería al lugar específico en el que se realizaban las entrevistas, no se refería al país entero o a la ciudad donde tenían lugar, por lo cual la medida, además de no constituir información sustancial, se limitaba a lo estrictamente necesario ${ }^{150}$.

\section{PRINCIPALES INCONVENIENTES Y POSIBLES SOLUCIONES EN MATERIA DE RESTRICCIONES AL DEBER DE DIVULGACIÓN DE INFORMACIÓN EN EL PROCESO PENAL INTERNACIONAL}

\subsection{Sobre los procedimientos 'ex parte' y la necesidad de crear nuevos cargos dentro de la CPI}

Las audiencias ex parte implican varios problemas para el derecho de defensa y la imparcialidad del juez que pueden tener un impacto significativo en el resultado final del proceso. En primer lugar, este tipo de procedimientos niegan cualquier posibilidad de ejercer una efectiva contradicción, como se vio, puede suceder que, incluso, la defensa no se entere siquiera de la existencia de esas audiencias. Al no existir esta posibilidad, hay una mayor probabilidad para el fiscal de convencer al juez sobre la excepción al deber de revelación, limitando el acceso a información relevante para la defensa. En una audiencia en la cual participan únicamente la fiscalía y el juez, sólo una visión de la situación se presenta ante los ojos del juzgador, la cual, al no tener un contrapeso, puede imponerse con facilidad.

En segundo lugar, el juez que decide la excepción al descubrimiento probatorio posteriormente participa en la confirmación de cargos o el juicio. La providencia que ordena la excepción, como se vio, se determina con base en el riesgo de peligro que supone el descubrimiento al acusado. Esto implica un cambio de perspectiva sobre el sospechoso pues, por ejemplo, en ocasiones se ha argumentado que la divulgación de determinada información puede acarrear que el procesado ejerza retaliaciones en contra de víctimas o testigos. Esto, sin dudas, puede desembocar en una grave parcialidad $^{151}$ del juez que posteriormente tendrá que decidir si el caso debe ser llevado

150 Ídem.

151 Posiblemente sea esta la razón por la cual, en ocasiones, el juez que decidía sobre la excepción al deber de información posteriormente era el único que en la confirmación de cargos estaba a favor de esta y se apartaba de la opinión mayoritaria de los magistrados que decidían no confirmar los cargos. A manera de ejemplo véase International Criminal Court. Prosecutor v. Abdallah Banda Abakaer Nourain and Saleh Mohammed Jerbo Jamus. "First Decision on the Prosecutor's Request for Redactions", ICC-02/05-03/09. 29 July 2010; y International Criminal Court. Prosecutor 
a juicio o, incluso, si el procesado será condenado o no, poniendo en duda la efectividad de su derecho al fair trial.

En tercer lugar, ya que no se tiene acceso al contenido de esa audiencia, o se tiene, pero con redacciones, la controversia o apelación de esa decisión es casi imposible pues no existe forma de contra argumentar lo desconocido, de ahí que el levantamiento de esas medidas dependerá, en últimas, de la voluntad del fiscal o del juez.

Dadas las razones anteriores, un efectivo contradictorio es difícilmente alcanzable en este tipo de procedimientos, lo cual sitúa a la defensa en una desventaja sustancial vis à vis la fiscalía, de ahí que en este texto se propongan dos soluciones para hacer frente a los problemas sobre el derecho de defensa e imparcialidad que acaban de ser mencionados: la creación de un representante especial de la defensa para los procedimientos ex parte y la creación de un juez de control de garantías.

El establecimiento de un representante especial de la defensa para las audiencias ex parte solventa los problemas relacionados con el derecho de defensa y el principio de contradicción. Su presencia establecerá un diálogo real con la contraparte de manera tal que el juez pueda tomar una decisión más razonada, basada en argumentos y contraargumentos y no simplemente en una visión parcializada de la situación. Así mismo, teniendo pleno conocimiento de las razones que se aducen para tomar la decisión en dicha audiencia, podrá posteriormente apelarla o ejercer una revisión constante, de manera tal que cuando exista un cambio de circunstancias, pueda solicitar el levantamiento de la restricción y no dependerá, exclusivamente, de la buena voluntad del fiscal o del juez. Claramente, en caso de proceder a la no divulgación, será necesario proscribirle al representante de la defensa la revelación de lo sucedido en audiencia. De esta manera se optimiza tanto la protección de los derechos del acusado como la garantía de los intereses que juegan en contra.

Finalmente, la creación de un juez de control de garantías deviene en un remedio efectivo para los posibles inconvenientes de imparcialidad. Este juzgador es el juez constitucional por excelencia, garante de los derechos fundamentales, quien tiene a su cargo la ponderación y armonización de derechos en conflicto ${ }^{152}$. El juez de control de garantías promoverá el respeto por los derechos y las libertades individuales y al mismo tiempo garantizará la eficacia de la investigación penal, será independiente

v. Abdallah Banda Abakaer Nourain and Saleh Mohammed Jerbo Jamus. "Corrigendum on the "Decision on the confirmation of Charges", ICC-02/05-03/09. 7 March 2011.

152 Corte Constitucional de Colombia. Sentencia C-210 de 2007, MP: Marco Gerardo Monroy Cabra. Véase también Corte Constitucional de Colombia. Sentencia C-591 de 2005, MP: Clara Inés Vargas Hernández; Corte Constitucional de Colombia. Sentencia C-873 de 2003, MP: Manuel José Cepeda Espinosa. A profundo detalle sobre el juez de control de garantías, Oscar Julián Guerrero Peralta. El control de garantías como construcción de una función jurisdiccional, Bogotá, Escuela Judicial "Rodrigo Lara Bonilla", 2006. 
e imparcial, decidirá únicamente en Derecho y su función principal será velar por la vigencia de los derechos del procesado. De esta manera, no sólo se evita que quien toma la decisión de restringir los derechos del sospechoso sea también el que posteriormente decida la determinación final en la confirmación de cargos o el juicio, sino que las decisiones del juez de control de garantías gozarán de mayor exactitud y legitimidad al ser un juez especializado en este ámbito.

\subsection{Sobre la inconsistencia e insuficiencia en la argumentación jurídica de las salas de la CPI y la necesidad de reglamentar la doctrina elaborada por la Corte}

Dos situaciones problemáticas se constatan en la jurisprudencia de la CPI. La primera consiste en que en ocasiones se emplea la doctrina elaborada por la Corte ${ }^{153}$ y en otras no se lleva a cabo sino que se procede a decretar la excepción sin un análisis de los requisitos que se estudiaron previamente ${ }^{154}$. La segunda es que aquellos casos en los que sí se ha hecho referencia a los requisitos planteados por la Corte, algunas veces la argumentación esgrimida es marcadamente detallada ${ }^{155}$ y, en otras, los razonamientos de las salas son demasiado simples, carecen de toda profundidad y la mención a los requisitos es una actividad apenas mecánica sin ningún ejercicio de reflexión ${ }^{156}$.

Las situaciones descritas plantean problemas de seguridad jurídica, son escenarios en los cuales no existe verdadera administración de justicia, donde los jueces incumplen con la básica obligación de motivar sus decisiones, lo cual desemboca en una ausencia de predictibilidad y coherencia que deja ver la calidad de justicia que se ejerce, y se genera una suerte de deslegitimación de la jurisdicción internacional que afecta la confianza de los Estados, y bien se sabe que en esa confianza se soporta la estructura de la CPI.

153 A manera de ejemplo, puede consultarse International Criminal Court. Prosecutor v. Germain Katanga. "Judgment on the Appeal of the Prosecutor Against the Decision of Pre-Trial Chamber I entitled 'First Decision on the Prosecution Request for Authorisation to Redact Witness Statements"'. 13 May 2008, ICC-01/04-01/07 (OA).

154 A manera de ejemplo, puede consultarse International Criminal Court. Prosecutor v. Abdallah Banda Abakaer Nourain and Saleh Mohammed Jerbo Jamus. "Decision on the Defence's Request for Disclosure of Documents in the Possession of the Office of the Prosecutor", ICC-02/05-03/09. 23 January 2013; International Criminal Court. Prosecutor v. Abdallah Banda Abakaer Nourain and Saleh Mohammed Jerbo Jamus. "Judgment on the appeal of Mr. Abdallah Banda Abakaer Nourain and Saleh Mohammed Jerbo Jamus against the decision of Trial Chamber IV of 23 January 2013 entitled 'Decision on the Defense's request for Disclosure of Documents in the Possession of the Office of the Prosecutor'”, ICC-02/05-03/09 OA 4. 28 August 2013.

155 A manera de ejemplo, consultar International Criminal Court. Prosecutor v. Callixte Mbarushimana. "Decision on the Prosecution's Application for Redactions pursuant to Rule 81 (2) and Rule 81 (4)", ICC-01/04-01/10. 20 May 2011.

156 A manera de ejemplo, consultar International Criminal Court. Prosecutor v. Callixte Mbarushimana. "Decision on Matters Raised at the Status Conference of 14 February 2011”, ICC-01/04-01/10, 2011. 
La solución que desde este texto se plantea es la reglamentación del esquema metodológico elaborado por la jurisprudencia de la Corte, de tal forma que se obligue a los jueces a señalar paso a paso el proceso ponderativo mediante el cual llegan a su decisión, que se haga imperativo el uso de esta herramienta analítica de manera que sea plenamente constatable en todos los casos el camino seguido para la adopción de la decisión final. Si bien es cierto que esta medida podría limitar la autonomía judicial, puede establecerse una excepción que consista en la posibilidad del juez de apartarse de esta doctrina si justifica razonadamente su actuar y, en todo caso, expresando en su decisión de manera explícita las razones que autorizan o niegan el no descubrimiento bajo la nueva metodología que se adopte.

\section{3. ¿El problema del lenguaje en el escenario del proceso penal internacional motiva la adopción de un derecho procesal penal del enemigo? La necesidad de un cambio de terminología ${ }^{157}$}

El proceso penal internacional se desenvuelve en un escenario que incrementa la probabilidad de concebir al sospechoso como enemigo ${ }^{158}$. El tipo de delitos que se investigan, las complicadas circunstancias de los países en donde los hechos delictivos tienen lugar, el uso del miedo como instrumento de manipulación y condicionante de las políticas de seguridad, la ausencia de cooperación de los Estados y la presión de la comunidad internacional por resultados ${ }^{159}$, la posibilidad de camuflar ideologías dado el relativismo de ciertas instituciones jurídicas y el lenguaje propio del proceso penal que hace referencia a contextos adversariales, partes en contienda y armas, entre otros, son algunos de los factores que pueden contribuir a un cambio de perspectiva sobre el acusado que desemboque en la primacía de la efectividad del ejercicio de la acción penal sobre y a expensas de las garantías y derechos fundamentales de este.

Esta situación es tan evidente que el mismo Jakobs señala que el derecho penal y procesal penal del enemigo se puede encontrar de manera clara y palpable en la jurisdicción internacional. Allí, arguye, la pena tiene la función de crear la vigencia de la norma y no de mantenerla ${ }^{160}$ pues las extremas vulneraciones a derechos humanos que se juzgan tienen lugar en aquellos sitios donde estos derechos no están

157 Véase en mayor detalle Simón Moratto. "El lenguaje en el Procedimiento Penal ¿Motiva la adopción de un Derecho Procesal Penal del Enemigo?”, en Memorias del Tercer Encuentro Internacional de Estudios Críticos de las Transiciones Políticas, Bogotá, Universidad de los Andes, 2019.

158 Sobre el concepto de enemigo, véase Günther Jakobs. "Derecho Penal del Ciudadano y Derecho Penal del Enemigo", en Manuel Cancio Meliá y Günther Jakobs. Derecho Penal del Enemigo. Madrid, Civitas, 2003, pp. 19-56.

159 Lo que puede resultar en una suerte de "gerencialismo". Sobre este concepto en el ámbito del Derecho Penal véase Jesús-María Silva Sánchez. La Expansión del Derecho Penal, 3. a ed., Madrid, Montevideo, Edisofer, B de F, 2011,p. 74. 
establecidos en el sentido de que sean "respetados a grandes rasgos"161. Reitera que no se trata de mantener un estado comunitario-legal sino de establecerlo y la situación previa a ese establecimiento es el estado de naturaleza, en el cual no hay personalidad. Es más: los autores de esas vulneraciones no ofrecen siquiera la seguridad suficiente para ser personas, por lo que frente a ellos "está permitido todo lo que sea necesario para asegurar el ámbito "comunitario-legal"'162 (énfasis propio). Argumenta que para ese establecimiento es necesario castigar a quien vulnere los derechos humanos mediante una sanción que, sin embargo, "no es una pena contra personas culpables, sino contra enemigos peligrosos"163. En resumen, ya que lo que se tiene es un estado de naturaleza y, dado que aquellos infractores no son personas, ninguna garantía jurídica es predicable de ellos, por lo que la ideología del enemigo, tanto en su faceta sustancial como procesal ${ }^{164}$, se puede manifestar en su máximo esplendor en el escenario del derecho penal internacional.

Todas y cada una de las anteriores situaciones ameritan una investigación propia que excede los límites de este texto. No obstante, es factible plantear aquí el inicio de una posible solución a uno de estos escenarios, concretamente, aquel en relación con el lenguaje que fortalece el desarrollo de la ideología del enemigo en el ámbito internacional.

Ese inicio consiste en un simple -pero con profundo significado- cambio de terminología sobre la igualdad entre las partes. Se propone abandonar el concepto de "armas" para hacer referencia a la igualdad procesal y en su lugar adoptar la noción de igualdad de medios y oportunidades. De esta manera se desecha un término que evoca, promueve y contribuye a la concepción y el reforzamiento de la ideología del enemigo sin necesidad de desnaturalizar ni sacrificar el significado y desarrollo que connota la garantía de igualdad procesal.

Este cambio de terminología deviene relevante en la medida en que el lenguaje condiciona la forma en la que las personas ven e interactúan con el mundo, construye una perspectiva con base en la cual se desarrollan los vínculos interpersonales en una sociedad, dentro de los cuales están las relaciones jurídicas. Este condicionamiento es tan fuerte que no por nada Wittgenstein, el gran filósofo del lenguaje, sostenía que los límites del lenguaje son los límites del mundo ${ }^{165}$.

161 Ibídem, p. 52.

162 Ibídem, p. 54

163 Ibídem, p. 55.

164 Sobre la faceta procedimental del derecho penal del enemigo, además de los trabajos del profesor Jakobs, también conviene consultar Francisco Muñoz Conde. De las prohibiciones probatorias al Derecho Procesal Penal del Enemigo, Buenos Aires, Hammurabi, 2008.

165 Ludwig Wittgenstein. Tractatus logico-philosophicus e Investigaciones filosóficas, Madrid, Gredos, 2009. 
Finalmente, como se dijo, estas son problemáticas que necesitan discusión y años de investigación si se quieren generar cambios significativos. Especialmente en el ámbito de la propuesta que aquí se hace, se requieren trabajos especializados en filosofía del lenguaje jurídico. El panorama no es del todo oscuro pues las soluciones a estos inconvenientes, aunque demandan un trabajo arduo, son perfectamente posibles como anteriormente se vislumbró. Aquí se dejan establecidas bases que pueden constituir el punto de partida de nuevas investigaciones que hagan viable un ejercicio más legítimo de la administración de justicia.

\section{CONCLUSIONES}

Primera. El principio de proporcionalidad no tiene referencia explícita en el cuerpo normativo de la CPI, sin embargo, la jurisprudencia ha encontrado su fundamento en el artículo 81 (4) y la regla 68 (1) de las RPP y a partir de allí ha desarrollado su doctrina. Por otro lado, el descubrimiento probatorio, es decir, la revelación de medios de prueba a la contraparte, de acuerdo con las reglas procesales, en la CPI encuentra su fundamento normativo en el artículo 68 del Estatuto y en la sección II del capítulo IV de las RPP, además de la regla 121.

Segunda. El principio de proporcionalidad es una herramienta analítica a través de la cual es posible determinar si es razonable o no la procedencia de restricciones al descubrimiento probatorio.

Tercera. Se constató que la función del principio de proporcionalidad en el marco de las restricciones al descubrimiento probatorio se desarrolla en el proceso ponderativo, el cual se compone de una serie de requisitos elaborados por la jurisprudencia de la CPI que deben satisfacerse para poder ordenar las restricciones al deber de divulgación. Estos requerimientos son: (1) la existencia de un riesgo objetivamente justificable; (2) la infactibilidad o insuficiencia de medidas menos restrictivas; y (3) la evaluación acerca de si las medidas que se buscan vulneran o no el derecho del acusado a un juicio justo e imparcial; y la obligación de la Sala, con ayuda del fiscal, de revisar periódicamente la decisión que autoriza el no descubrimiento.

Cuarta. La reseñada doctrina elaborada por la Corte presenta varios inconvenientes, a saber: (1) la parcialidad y las afectaciones al ejercicio de una efectiva defensa y controversia de las decisiones tomadas en procedimientos ex parte; (2) la inconsistencia e insuficiencia en la argumentación jurídica de la Corte; y (3) el problema del lenguaje en el escenario del proceso penal internacional y la necesidad de un cambio de terminología.

Quinta. Frente a las anteriores problemáticas, se establecieron propuestas de posibles soluciones para cada una de ellas, respectivamente: (1) la creación de un representante especial de la defensa que participe en las audiencias ex parte y la creación de un juez de control de garantías; (2) la reglamentación de la doctrina elaborada por 
la Corte para autorizar el no descubrimiento de información; y (3) el abandono del término "armas" para hacer referencia a la igualdad procesal y, en su lugar, optar por el concepto de igualdad de medios y oportunidades.

\section{REFERENCIAS}

\section{Doctrina}

Alexy, Robert. "Sobre los derechos constitucionales a protección”, en Robert Alexy, José Bastida Freijedo, Juan Antonio García Amado, Alfonso Jaime García Figueroa, Luis Hierro Sánchez-Pescador, Josep Joan Moreso, C. Pardo, Gregorio Peces-Barba y Ernesto Valdés Garzón. Derechos sociales y ponderación, Madrid, Fundación Coloquio Jurídico Europeo, 2009.

Alexy, Robert. Teoría de los derechos fundamentales, Madrid, Centro de Estudios Políticos y Constitucionales, 2007.

Ambos, Kai. "Confidential investigations (article 54 (3)(E) ICC Statute) vs. Disclosure obligations: The Lubanga Case and National Law", en New Criminal Law Review: An International and Interdisciplinary Journal, Berkeley, University of California Press, Fall 2009, vol. 12, n. ${ }^{\circ}$ 4, pp. 543-568, disponible en [https://www.legal-tools. org/doc/c76c63/pdf/] (consulta: 7 de julio de 2019).

Ambos, Kai. "The First judgement of the International Criminal Court (Prosecutor v. Lubanga): A comprehensive analysis of the legal issues", en International Criminal Law Review, Leiden, Brill, 2012, vol. 12, pp. 115-153, disponible en [https://papers. ssrn.com/sol3/papers.cfm?abstract_id=2030751] (consulta: 7 de julio de 2019).

Ambos, Kai. Treatise on International Criminal Law, Oxford, Oxford University Press, 2013.

Barak, Aharon. Proporcionalidad: los derechos fundamentales y sus restricciones, Lima, Palestra Editores, 2017.

Bernal Cuéllar, Jaime y Eduardo Montealegre Lynett. El proceso penal, tomo I: Fundamentos constitucionales y teoría general, Bogotá, Universidad Externado de Colombia, 2013.

Bernal Cuéllar, Jaime y Eduardo Montealegre Lynett. El proceso penal, tomo II: Estructura y garantías procesales, 6. edición, Bogotá, Universidad Externado de Colombia, 2013.

Bernal Pulido, Carlos. El principio de proporcionalidad y los derechos fundamentales, Madrid, Centro de Estudios Políticos y Constitucionales, 2007. 
Brady, Helen. "Disclosure of Evidence”, en Roy S. Lee. The International Criminal Court: Elements of Crimes and Rules of Procedure and Evidence, Ardsley, Transnational Publishers, 2001.

Campbell Black, Henry. Black's Law Dictionary. Definitions of the Terms and Phrases

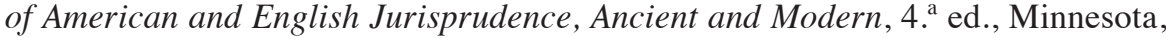
West Publishing Company, 1968.

Chang-Jung Yang, Alice. The Prosecution's Duty of Disclosure before International Criminal Tribunals (doctoral thesis), London, Brunel University-Brunel Law School, 2016.

Cianello, Michele. "Disclosure before the ICC: The emergence of a new form of policies implementation system in International Criminal Justice?", en International Criminal Law Review, Leiden, Brill, 2010, col. 10, pp. 23-42.

Fedorova, Masha. The Principle of Equality of Arms in International Criminal Proceedings, Cambridge, Intersentia, 2013.

Fiori, Brando. Disclosure of Information in Criminal Proceedings: A Comparative Analysis of national and international criminal procedural systems and human rights law, Oisterwijk, Wolf Legal Publishers, 2015.

Guerrero Peralta, Oscar Julián. El control de garantías como construcción de una función jurisdiccional, Bogotá, Escuela Judicial "Rodrigo Lara Bonilla”, 2006.

Heinze, Alexander. International Criminal Procedure and Disclosure: an attempt to better understand and regulate disclosure and communication at the ICC on the basis of a comprehensive and comparative theory of criminal procedure, Berlin, Duncker and Humblot GmbH, 2014.

Jakobs, Günther. "Derecho Penal del Ciudadano y Derecho Penal del Enemigo", en Manuel Cancio Meliá y Günther Jakobs. Derecho Penal del Enemigo, Madrid, Civitas Ediciones, S. L. 2003.

Londoño Ayala, César Augusto. Principio de proporcionalidad en el Derecho procesal penal, Bogotá, Ediciones Nueva Jurídica, 2009.

Meester, Karel de; Kelly Pitcher; Rod Rastan y Goran Sluiter. "Investigation, Coercive Measures, Arrest and Surrender", en Hakan Friman, Goran Sluiter, Suzannah Linton, Sergey Vasiliev y Salvatore Zappala (eds.). International Criminal Procedure. Principles and Rules, Oxford, Oxford University Press, 2013. 
Moratto, Simón. "El lenguaje en el procedimiento penal: ¿motiva la adopción de un derecho procesal penal del enemigo?", en Memorias del Tercer Encuentro Internacional de Estudios Críticos de las Transiciones Políticas, Bogotá, Universidad de los Andes, 2019.

Muñoz Conde, Francisco. De las prohibiciones probatorias al derecho procesal penal del enemigo, Buenos Aires, Hammurabi, 2008.

Safferling, Cristoph. International Criminal Procedure, Oxford, Oxford University Press, 2012.

Schuon, Christine. International Criminal Procedure: A Clash of Legal Cultures, The Hague, T. M. C. Asser Press, 2010.

Silva Sánchez, Jesús-María. La expansión del Derecho Penal, 3. a ed., Madrid, Montevideo, Edisofer, B de F, 2011.

Wittgenstein, Ludwig. Tractatus logico-philosophicus e investigaciones filosóficas, Madrid, Gredos, D. L. 2009.

\section{Normativa}

Corte Penal Internacional. Asamblea de los Estados Partes. Resolución ICC-ASP/4/ Res.1, por la que se aprueba el Código de Conducta Profesional de los Abogados, 2 de diciembre de 2005.

Corte Penal Internacional. Magistrados de la Corte, Quinta sesión plenaria, La Haya, 17-28 de mayo de 2004. Documentos oficiales de la Corte Penal Internacional. ICC-BD/01-01-04. Reglamento de la Corte, 26 de mayo de 2004.

Corte Penal Internacional. Magistrados de la Corte, Quinta sesión plenaria, La Haya, 17-28 de mayo de 2004. Documentos oficiales de la Corte Penal Internacional. ICC-BD/01-01-04. Reglamento de la Corte, 26 de mayo de 2004.

Corte Penal Internacional. Presidencia de la Corte Penal Internacional. Documentos oficiales de la Corte Penal Internacional. ICC-BD/03-01-06. Reglamento de la Secretaría, 6 de marzo de 2006.

\section{Jurisprudencia relevante}

Corte Constitucional de Colombia. Sentencia C-1194 de 2005, MP: Marco Gerardo Monroy Cabra. 
Corte Constitucional de Colombia. Sentencia C-210 de 2007, MP: Marco Gerardo Monroy Cabra.

Corte Constitucional de Colombia. Sentencia C-591 de 2005, MP: Clara Inés Vargas Hernández.

Corte Constitucional de Colombia. Sentencia C-873 de 2003, MP: Manuel José Cepeda Espinosa.

International Criminal Tribunal for the Former Yugoslavia (Trial Chamber) Prosecutor v. Milomir Stakic. Order to the Registry of the Tribunal to provide documents. 5 July 2002.

International Criminal Court. Prosecutor v. Thomas Lubanga Dyilo. "Decision on the Final System of Disclosure and the Establishment of a Timetable", ICC-01/04-01/06. 15 May 2006.

International Criminal Court. Prosecutor v. Thomas Lubanga Dyilo. "Decision Establishing General Principles Governing Applications to Restrict Disclosure pursuant to Rule 81 (2) and (4) of the Rules of Procedure and Evidence", ICC-01/04-01/06. 19 May 2006.

International Criminal Court. Prosecutor v. Thomas Lubanga Dyilo. "Judgment on the Appeal of Mr. Thomas Lubanga Dyilo against the Decision of Pre-Trial Chamber I entitled 'First Decision on the Prosecution Requests and Amended Requests for Redactions under Rule 81"”, ICC-01/04-01/06 (OA 5). 14 December 2006.

International Criminal Court. Prosecutor v. Thomas Lubanga Dyilo. "Judgment on the Appeal of Mr. Thomas Lubanga Dyilo against the Decision of Pre-Trial Chamber I entitled 'First Decision on the Prosecution Requests and Amended Requests for Redactions under Rule 81'”, ICC-01/04-01/06-773. 14 December 2006.

International Criminal Court. Prosecutor v. Dominic Ongwen. "Decision on Legal Representation, Appointment of Counsel for the Defence, Protective Measures and Time-limit for Submission of Observations on Applications a/0010/06, a/0064/06, to a/0070/06, a/0081/06 to a/0104/06 and a/0111/06 to a/0127/06", ICC-02/04-01/05. 1 February 2007.

International Criminal Court. Prosecutor v. Thomas Lubanga Dyilo. "Decision on the Disclosure by the Defence”, ICC-01/04-01/06. 20 March 2008.

International Criminal Court. Prosecutor v. Germain Katanga and Mathieu Ngudjolo Chui. "Sixth Decision on the Prosecution Request for Authorisation to Redact the Interviews Transcripts of Witness 238", ICC-01/04-01/07. 21 April 2008. 
International Criminal Court Prosecutor v. Germain Katanga. "Judgment on the Appeal of the Prosecutor Against the Decision of Pre-Trial Chamber I entitled 'First Decision on the Prosecution Request for Authorisation to Redact Witness Statements", ICC-01/04-01/07 (OA). 13 May 2008.

International Criminal Court. Prosecutor v. Thomas Lubanga Dyilo. "Decision on the consequences of non-disclosure of exculpatory materials covered by article 54 (3) (e) agreements and the application to stay the prosecution of the accused, together with certain other issues raised at the status conference on 10 June 2008", ICC-01/0401/06. 13 June 2008.

International Criminal Court. Prosecutor v. Thomas Lubanga Dyilo. "Judgment on the appeals of The prosecutor and The defence against Trial Chamber I's Decision on Victims' participation of 18 January 2008", ICC-01/04-01/06-1432. 11 July 2008.

International Criminal Court. Prosecutor v. Jean-Pierre Bemba Gombo. "Public redacted version of ICC-01/05-01/08-48-US-Exp Decision Concerning the Prosecutor's Proposals for Redactions", ICC-01/05-01/08. 23 July 2008.

International Criminal Court. Prosecutor v. Jean Pierre Bemba Gombo. "Decision on the evidence disclosure system and setting a Timetable for disclosure between the parties", ICC-01/05-01/08. 31 July 2008.

International Criminal Court. Prosecutor v. Jean-Pierre Bemba Gombo. "Public redacted version of ICC-01/05-01/08-135-Conf Second Decision on the Prosecutor's Request for Redactions", ICC-01/05-01/08. 1 October 2008.

International Criminal Court. Prosecutor v. Thomas Lubanga Dyilo. "Judgement on the appeal of the prosecutor against the decision of Trial Chamber I entitled 'Decision on the consequences of non-disclosure of exculpatory materials covered by article 54 (3) (e) agreements and the application to stay the prosecution of the accused, together with other issues raised at the status conference on 10 June 2008'”, ICC-01/04-01/06. 21 October 2008.

International Criminal Court. Prosecutor v. Jean Pierre-Bemba Gombo. "Public redacted version of ICC-01/05-01/08-215-Conf Third Decision on the Prosecutor's Requests for Redactions and Related Request for the Regulation of Contacts of JeanPierre Bemba Gombo, ICC-01/05-01/08. 6 November 2008.

International Criminal Court. Prosecutor v. Bemba. "Judgment on the Appeal of Mr. Jean-Pierre Bemba Gombo against the Decision of Pre-Trial Chamber III entitled 'Decision on Application for Interim Release'”, ICC-01/05-01/08-323. 16 December 2008 . 
International Criminal Court. Prosecutor v. Bahar Idriss Abu Garda. "Public Redacted Version of the 'First Decision on the prosecution's Request for Redactions' issued on 14 August 2009”, ICC-02/05-02/09. 20 August 2009.

International Criminal Tribunal for Rwanda. Prosecutor v. Karemera et al., Trial Chamber III. "Decision on motion for Disclosure of Medical Information and Extension of Time", ICTR-98-44-T. 28 August 2009.

International Criminal Court. Prosecutor v. Abdallah Banda Abakaer Nourain and Saleh Mohammed Jerbo Jamus. "First Decision on the Prosecutor's Request for Redactions", ICC-02/05-03/09. 29 July 2010.

International Criminal Court. Prosecutorv. Germain Katanga and Mathieu Ngudjolo Chui. "Decision on the 'prosecution's application concerning disclosure by the defence pursuant to rules 78 and 79 (4)'”, ICC-01/04-01/07. 14 September 2010.

International Criminal Court. Prosecutor v. Abdallah Banda Abakaer Nourain and Saleh Mohammed Jerbo Jamus. "Corrigendum on the "Decision on the confirmation of Charges", ICC-02/05-03/09. 7 March 2011.

International Criminal Court. Prosecutor v. Callixte Mbarushimana. "Decision on Matters Raised at the Status Conference of 14 February 2011", ICC-01/04-01/10. 21 March 2011.

International Criminal Court. Prosecutor v. Callixte Mbarushimana. "Decision on Issues Relating to Disclosure", ICC-01/04-01/10. 30 March 2011.

International Criminal Court. Prosecutor v. William Samoei Ruto and Joshua Arap Sang. "Decision Setting the Regime for Evidence Disclosure and Other Related Matters", ICC-01/09-01/11. 6 April 2011.

International Criminal Court. Prosecutor v. Callixte Mbarushimana. "Decision on the Prosecution's Application for Redactions pursuant to Rule 81 (2) and Rule 81 (4)", ICC-01/04-01/10. 20 May 2011.

International Criminal Court. Prosecutor v. Callixte Mbarushimana. "Decision on the Review of Potentially Privileged Material”, ICC-01/04-01/10. 15 June 2011.

International Criminal Court. Prosecutor v. Francis Kirimi Muthaura, Uhuru Muigai Kenyatta and Mohammed Hussein Ali. "Decision on the Confidential Redacted Version of the Article 58 Application”, ICC-01/09-02/11. 22 July 2011. 
International Criminal Court. Prosecutor v. Callixte Mbarushimana. "Decision on Prosecution's Application for Authorisation to Disclose a Document received pursuant Article 54 (3) (e) in redacted form”, ICC-01/04-01/10. 4 August 2011.

International Criminal Court. Prosecutor v. Abdallah Banda Abakaer Nourain and Saleh Mohammed Jerbo Jamus. "Decision on Article 54 (3) (e) documents", ICC02/05-03/09. 23 November 2011.

International Criminal Court. Prosecutor v. Jean Pierre Bemba Gombo. "Decision on the Defence Disclosure and Related Issues", ICC-01/05-01/08. 24 February 2012.

International Criminal Court. Prosecutor v. Francis Kirimi Muthaura and Uhuru Muigai Kenyatta. "Decision on the Protocol Establishing a Redaction Regime", ICC-01/09-02/11. 27 September 2012.

International Criminal Court. Prosecutor v. Abdallah Banda Abakaer Nourain and Saleh Mohammed Jerbo Jamus. "Decision on the Defence Request for a Temporary Stay of Proceedings", ICC-02/05-03/09. 26 October 2012.

International Criminal Court. Prosecutor v. William Samoei Ruto and Joshua Arap Sang. "Decision on Prosecution Application to Redact Investigator's Identifying Information and to Vary the Redaction Protocol", ICC-01/09-01/11.21 December 2012.

International Criminal Court. Prosecutor v. William Samoei Ruto and Joshua Arap Sang. "Confidential Redacted Version of 'Decision on First Prosecution Application for Delayed Disclosure of Witness Identities"”, ICC-01/09-01/11. 4 January 2013.

International Criminal Court. Prosecutor v. Abdallah Banda Abakaer Nourain and Saleh Mohammed Jerbo Jamus. "Decision on the Defence's Request for Disclosure of Documents in the Possession of the Office of the Prosecutor", ICC-02/05-03/09. 23 January 2013.

International Criminal Court. Prosecutor v. William Samoei Ruto and Joshua Arap Sang. "Decision on the Prosecution's Application for Authorisation to Maintain Certain Redactions”, ICC-01/09-01/11. 23 April 2013.

International Criminal Court. Prosecutor v. William Samoei Ruto and Joshua Arap Sang. "Confidential Redacted Version of the 'Decision on the Prosecution's Renewed Request for Delayed Disclosure of Identity of Witness 534'”, ICC-01/09-011. 2 May 2013.

International Criminal Court. Prosecutor v. Saif Al-Islam Gaddafi and Abdullah AlSenussi. "Decision on the Prosecution's Request for Redactions pursuant to Rule 81 (2)", ICC-01/11-01/11. 16 August 2013. 
International Criminal Court. Prosecutor v. Abdallah Banda Abakaer Nourain and Saleh Mohammed Jerbo Jamus. "Judgment on the appeal of Mr. Abdallah Banda Abakaer Nourain and Saleh Mohammed Jerbo Jamus against the decision of Trial Chamber IV of 23 January 2013 entitled 'Decision on the Defense's request for Disclosure of Documents in the Possession of the Office of the Prosecutor'", ICC02/05-03/09 OA 4. 28 August 2013.

International Criminal Court. Prosecutor v. William Samoei Ruto and Joshua Arap Sang. "Decision on Disclosure of Information related to Prosecution Intermediaries", ICC-01/09-01/11. 4 September 2013.

International Criminal Court. Prosecutor v. Jean-Pierre Bemba Gombo, Aimé Kilolo Musamba, Jean-Jacques Mangenda Kabongo, Fidelé Babala Wandu and Narcisse Arido. "Decision on the 'Prosecution's Application for Redactions pursuant to Rules 81 (2) and 81 (4) of the Rules of Procedure and Evidence'. Dated 6 June 2014”, ICC-01/05-01/13. 17 June 2014.

International Criminal Court. Prosecutor v. Dominic Ongwen. "Decision Setting the Regime for Evidence Disclosure and Other Related Matters", ICC-02/04-01/15. 27 February 2015.

International Criminal Court. Prosecutor v. Dominic Ongwen. "Decision Concerning the Redaction and Transmission of Documents to the Defence", ICC-02/04-01/15. 15 April 2015.

International Criminal Court. Prosecutor v. Ahmad Al Faqi Al Mahdi. "Decision on Issues Related to Disclosure and Exceptions thereto", ICC-01/12-01/15. 30 September 2015 .

International Criminal Court. Prosecutor v. Ahmad Al Faqi Al Mahdi. "Decision on the Prosecutor's Request for Redactions", ICC-01/12-01/15. 8 December 2015.

International Criminal Court. Prosecutor v. Dominic Ongwen. "Decision on the Prosecutor's Request for Authorisation of non-standard Redactions", ICC-02/04-01/15. 23 December 2015.

International Criminal Court. Prosecutor v. Dominic Ongwen. "Decision on the Prosecution's Application under Article 53 (3) (f) to Apply Redactions to Documents Obtained under Article 54 (3) (e)", ICC-02/04-01/15. 2 March 2016.

International Criminal Court. Prosecutor v. Francis Kimiri Muthaura and Uhuru Muigai Kenyatta. "Decision on the Prosecution's First Request for the Authorisation of Redactions, 13 December 2012, ICC-01/09-02/11-569-Conf”, ICC-01/09-02/11. 3 February 2017. 
International Criminal Court. Prosecutor v. Francis Kimiri Muthaura and Uhuru Muigai Kenyatta. "Decision on Second Prosecution Application for Delayed Disclosure of Witness Identities, 8 January 2013, ICC-01/09-02/11-593-Conf-Exp", ICC-01/09-02/11. 3 February 2017.

International Criminal Court. Prosecutor v. Francis Kirimi Muthaura and Uhuru Muigai Kenyatta. "Decision on the Prosecution's Application for Relief Pursuant to Decision 451, Rule 81 (2) and Regulation 35, 9 January 2013, ICC-01/09-02/11595-Conf", ICC-01/09-02/11. 3 February 2017.

International Criminal Court. Prosecutor v. Al Hassan Ag Abdoul Aziz Ag Mohammed Ag Mahmoud. "Decision on the Evidence Disclosure Protocol and Other Related Matters", ICC-01/12-01/18. 16 May 2018.

International Criminal Court. Prosecutor v. Al Hassan Ag Abdoul Aziz Ag Mohammed Ag Mahmoud. "Decision on the Prosecution Motion for Authorization to File an Anonymous Summary Concerning Witness MLI-OTP-P-P0113”, ICC-01/12-01/18. 27 September 2018. 